\title{
Relative frequencies of supernovae types: dependence on host galaxy magnitude, galactocentric radius, and local metallicity
}

\author{
S. Boissier ${ }^{1}$ and N. Prantzos ${ }^{2}$ \\ ${ }^{1}$ Laboratoire d'Astrophysique de Marseille, OAMP, Université Aix-Marseille \& CNRS UMR6110, 38 rue Frédéric Joliot Curie, \\ 13388 Marseille Cedex 13, France \\ e-mail: samuel.boissier@oamp.fr \\ 2 Institut d'Astrophysique de Paris, UMR7095 CNRS, Univ. P. \& M. Curie, 98bis Bd. Arago, 75104 Paris, France \\ e-mail: prantzos@iap.fr
}

Received 27 October 2008 / Accepted 10 May 2009

ABSTRACT

\begin{abstract}
Context. Stellar evolution theory suggests that the relationship between number ratios of supernova (SN) types and metallicity can provide important information about the physical properties of the progenitor stars (e.g., mass, metallicity, rotation, binarity).

Aims. We investigate the metallicity dependence of the number ratios of various SN types, using a large sample of SN along with information about their radial position within, and magnitude of, their host galaxy.

Methods. We derive global galaxy gas-phase metallicities (using the well-known metallicity-luminosity relation) and local metallicities, i.e., at the position of the SN. In the latter case, we use the empirical fact that the metallicity gradients in disk galaxies are $\sim$ constant when expressed in $\mathrm{dex} / R_{25}$.

Results. We confirm a dependence of the $N(\mathrm{Ibc}) / N(\mathrm{II})$ ratio on metallicity. Single star models with rotation and binary star models with no rotation appear to reproduce equally well the metallicity dependence. The size of our sample does not allow significant conclusions about the $N(\mathrm{Ic}) / N(\mathrm{Ib})$ ratio. Finally, we find an unexpected metallicity dependence of the ratio of thermonuclear to core collapse supernovae, which we interpret in terms of the star formation properties of the host galaxies.
\end{abstract}

Key words. stars: supernovae: general

\section{Introduction}

The classification of supernovae in various types (II, Ib, Ic, Ia) is based on the presence of various features in their spectra such as the presence or absence of hydrogen, which distinguishes SNII from SNI, while within the SNI family, the presence of Si lines characterizes SNIa and the presence of He lines distinguishes SNIb from SNIc (see e.g, Hamuy 2002; Turatto 2003, and references therein). While SNIa are observed in all types of galaxies (ellipticals, irregulars, spirals), SNIb and Ic (collectively called SNIbc in this work including SN with types $\mathrm{Ib}$, Ic, and $\mathrm{Ib} / \mathrm{c}$ ), as well as SNII are only observed in star forming regions of spirals and irregulars. For that reason, they are assumed to originate in massive stars, as a result of the gravitational collapse of their $\mathrm{Fe}$ cores (gravitational SN or core collapse SN, CCSN in the following). Various schemes have been proposed to relate each one of those types to a progenitor star, either within the framework of single-star models (e.g., Heger et al. 2003; Maeder \& Meynet 2004) or binary star evolution (e.g., Eldridge et al. 2008).

A comprehensive summary of our understanding of the various CCSN types is provided in Fryer et al. (2007). Among the various factors affecting the surface chemical composition of a massive star at the time of its explosion, mass, metallicity, rotation, and binarity appear to play an important role, while the potential impact of others (e.g., magnetic fields) has not been sufficiently explored yet. The extent to which each one of the aforementioned factors (as well as their combined action) determines the mass lost by the star and its final surface composition has been the subject of intense theoretical and observational investigations. In the framework of single star models, it is believed that, for a given metallicity, the lowest mass progenitors of CCSN become SNII, stars more massive than some (yet poorly known) limit $M_{\mathrm{Ib}}$ produce $\mathrm{SNIb}$, and stars more massive than $M_{\text {Ic }}$ end their lives as SNIc. In principle, a decrease in metallicity increases both $M_{\mathrm{Ib}}$ and $M_{\mathrm{Ic}}$, but its effect may be compensated for by rotation, which mixes nucleosynthesis products from the core to the surface.

In an early attempt to determine empirically the role of metallicity in shaping the various CCSN types, Prantzos \& Boissier (2003, PB03 in the following) studied the relationship between the number ratio of SNIbc to SNII $N(\mathrm{Ibc}) / N(\mathrm{II})$ versus the typical metallicity of the host galaxy. In the absence of relevant metallicity measurements, they used the galaxian luminosity as a proxy for metallicity, taking advantage of the well-known metallicity-luminosity relation.

Assuming reasonable values for the slope of the IMF (in the -1.30 to -1.70 range), PB03 used the observed global ratio of $N(\mathrm{Ibc}) / N(\mathrm{II})$ to derive $M_{\mathrm{Ibc}} \sim 20-24 M_{\odot}$. This mass is comparable to the maximum mass for type II-P SN found by Smartt et al. (2009) in their volume-limited sample, $16.5 \pm 1.5 M_{\odot}$ for a Salpeter IMF (and up to $22 M_{\odot}$ for a steeper IMF). PB03 argued that this value is much lower than the one provided by nonrotating models of single star evolution, even at high metallicity (>30 $M_{\odot}$, e.g., Heger et al. 2003). They suggested instead that rotating models, such as the solar metallicity models of Meynet \& Maeder (2003) provide suitable alternative solutions. Furthermore, PB03 identified a correlation between $N(\mathrm{Ibc}) / N(\mathrm{II})$ and metallicity (albeit with large error bars) and attributed this 
to $M_{\mathrm{Ibc}}$ (the limiting mass between SNII and SNIbc) decreasing with metallicity $Z$. In the absence of relevant models at lower metallicities at that time, PB03 deduced the required $M_{\mathrm{Ibc}}=$ $f(Z)$ relationship, to fit the observational data (again, for reasonable values of the IMF slope). Subsequent models of low $Z$ massive rotating stars (Maeder \& Meynet 2004) confirmed those quantitative predictions relatively well. Similar observational results were obtained for the $N(\mathrm{Ibc}) / N(\mathrm{II})$ versus $Z$ relation by Prieto et al. (2008), who did not use the mass-metallicity relation but directly measured metallicities of a smaller sample of host galaxies from the SDSS database. In the meantime, Eldridge et al. (2008) proposed non-rotating models for binary star evolution, the results of which reproduce also satisfactorily the $N(\mathrm{Ibc}) / N(\mathrm{II})$ versus metallicity relation. We discuss those theoretical developments in Sect. 5.

In this work, we investigate the relation between metallicity and number ratios of various SN types with a much larger SN sample than PB03 (Sect. 2). We extend our study to the ratios of $N(\mathrm{Ic}) / N(\mathrm{Ib})$ and, for the first time, to $N(\mathrm{Ia}) / N(\mathrm{CC})$. Furthermore, we derive not only global (typical) galaxian metallicities (Sect. 3) but also local ones, i.e., at the position of the SN within its host galaxy. For that purpose, we use the fact that metallicity gradients appear to be constant when expressed in $\operatorname{dex} / R_{25}{ }^{1}$ (Sect. 4). We are thus able to establish statistically significant corelations with both global and local metallicity of $N(\mathrm{Ibc}) / N(\mathrm{II})$ and, somewhat surprisingly, of $N(\mathrm{Ia}) / N(\mathrm{CC})$. We discuss those findings in Sect. 5 and summarize our work in Sect. 6.

\section{The supernova sample}

We use a version of the Asiago Supernova Catalogue (presented in Barbon et al. 1999) to obtain information about a large number of supernovae and their host galaxies. This information concerns the SN type, magnitude (usually the discovery magnitude), and relative distance to the galactic center, the galaxy type, and various parameters, such as position angle, inclination, $R_{25}$ radius, and heliocentric radial velocity $V_{\mathrm{HEL}}$. For each galaxy, we use the LEDA database (Paturel et al. 2003) to obtain the $B$-band absolute magnitude $M_{B}$. In this list, we retain only galaxies with morphological types corresponding to spirals ( $\mathrm{S} 0$ to $\mathrm{Sd}$ ) and irregulars (Irr) because we are interested in the ratio of the various supernovae types occuring in star-forming galaxies.

Because of differences in their intrinsic luminosities, various supernovae types can be detected at different distances. In particular, SN Ia are intrinsically brighter than core collapse SN and can be detected further away. Figure 1 (three top panels) shows the SN magnitude as a function of $V_{\mathrm{HEL}}$ in our sample. We are aware that these SN magnitudes are not perfectly well determined, and not always comparable to each other (discovery versus peak magnitude, different photometric bands, no true unique distance) but, overall, they provide a rough idea of the event brightness. The curves indicate the average values and $1-\sigma$ standard deviation from them. They are obtained by computing statistics within the indicated bin size at many points along the $x$-axis; the distances between those points is less than the bin size, i.e., the points are not independent. We also include four independent points along the curves with error bars. The grey shaded area indicates the absolute magnitude for $\mathrm{SN}$ fainter than 19 mag, where very few SN are found. This value corresponds roughly to the limiting magnitude of $\mathrm{SN}$ surveys, especially the LOSS SN Survey (van den Bergh et al. 2005) providing a large

${ }_{1}$ The $R_{25}$ radius is the radius of the $25 \mathrm{mag} \operatorname{arcsec}^{-2} B$-band isophote.

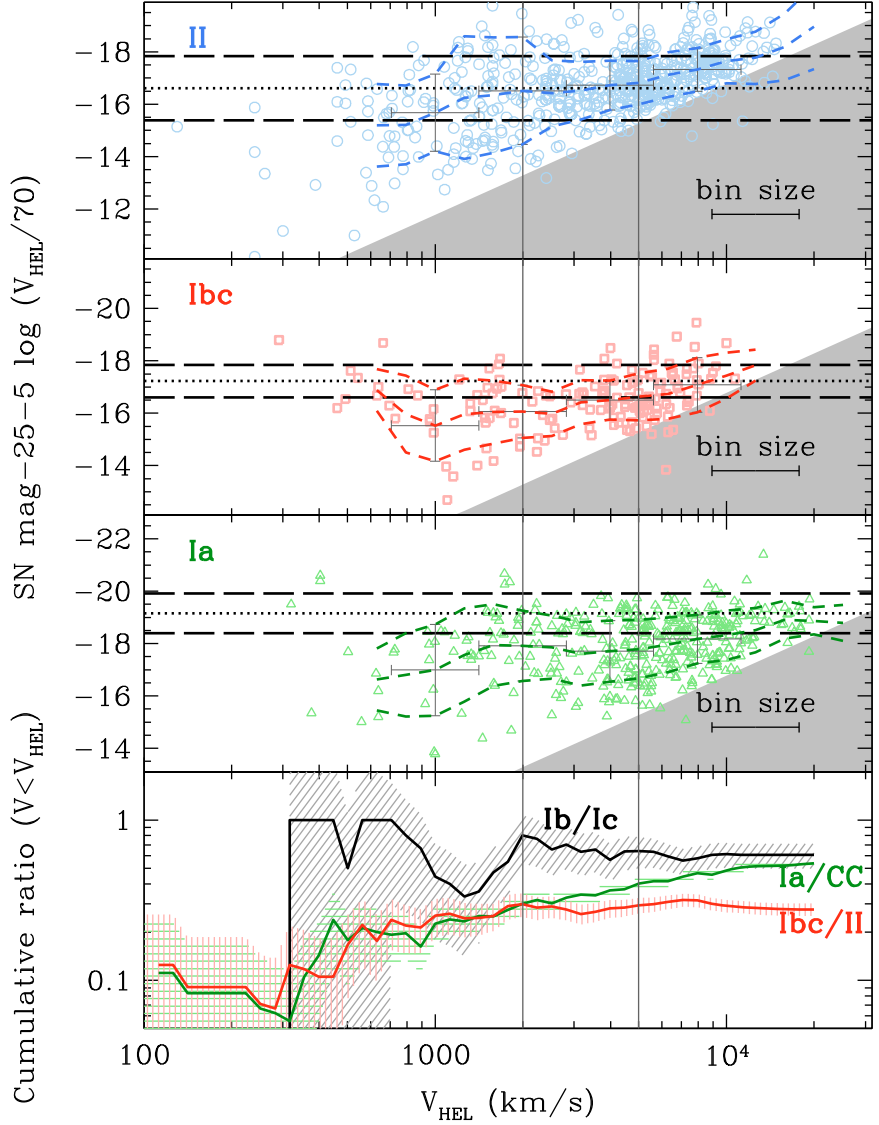

Fig. 1. Top three panels: supernovae absolute magnitude (adopting a basic Hubble's law with $H_{0}=70 \mathrm{~km} \mathrm{~s}^{-1} \mathrm{Mpc}^{-1}$ ) as a function of the heliocentric velocity, for SN of type II, Ibc, and Ia, respectively (from top to bottom). The curves show the average (solid) and $1 \sigma$ dispersion (dashed) within bins of size indicated in the bottom-right part of the figure (only points separated by a distance larger than the bin size are independent in these curves, see text). Independent points with error bars are also displayed in each panel. The dotted (and long-dashed) lines show the average magnitude (and its dispersion) given in Richardson et al. (2002). Almost no SN are found in the shaded area (apparent magnitudes fainter than 19) because it corresponds roughly to the limiting magnitude of the sample (see text). Bottom: cumulative ratios of SN types versus heliocentric velocity. All panels: the 2 vertical lines indicates the two velocity limits for which we present results: $5000 \mathrm{~km} \mathrm{~s}^{-1}$ (excluding distant galaxies for which we start missing large numbers of type II SN), and the more conservative $2000 \mathrm{~km} \mathrm{~s}^{-1}$.

number of SN each year. Although the Asiago catalogue does not represent a clearly defined sample, we can consider 19 to be our approximate limiting magnitude. Since the various SN types do not have identical absolute magnitudes, we must ensure that within the volume that we use, we do not miss some types while detecting others. To check this, we use the absolute magnitudes (and observation dispersion) given by Richardson et al. (2002) for various types: -16.61 (1.23) for SNII; -17.23 (0.62) for SNIbc; -19.16 (0.76) for Ia. These values are given in their Table 1 and correspond respectively to normal SN II-P, normal Ibc, and normal Ia. The most common type among SNII is II-P. Other types (IIn, IIL) are on average brighter, thus if there is no bias against II-P, there should be no bias against SNII either. We note that Richardson et al. (2002) find evidence for a bimodal distribution of the magnitudes of SNIbc, with a brighter component than the normal one. Here again, if we choose our sample in such a way as to include all normal SNIbc, we should 
Table 1. Size of the samples and ratios.

\begin{tabular}{lll}
\hline \hline SN & Larger number statistics & Conservative \\
Type & $V_{\mathrm{HEL}}<5000 \mathrm{~km} \mathrm{~s}^{-1}$ & $V_{\mathrm{HEL}}<2000 \mathrm{~km} \mathrm{~s}^{-1}$ \\
\hline $\mathrm{Ic}$ & $49(41)$ & $18(15)$ \\
$\mathrm{Ib}$ & $32(25)$ & $15(12)$ \\
$\mathrm{Ibc}$ & $98(79)$ & $43(36)$ \\
$\mathrm{II}$ & $318(239)$ & $142(96)$ \\
$\mathrm{Ia}$ & $166(132)$ & $56(42)$ \\
\hline$N(\mathrm{Ibc}) / N(\mathrm{II})$ & $0.31 \pm 0.04(0.06)$ & $0.30 \pm 0.05(0.06)$ \\
$N(\mathrm{Ic}) / N(\mathrm{Ib})$ & $1.53 \pm 0.35(0.30)$ & $1.20 \pm 0.42(0.24)$ \\
$N(\mathrm{Ia}) / N(\mathrm{CC})$ & $0.40 \pm 0.04(0.08)$ & $0.30 \pm 0.05(0.06)$ \\
\hline
\end{tabular}

Top part: the first number is the number of $\mathrm{SN}$ of a given type for which at least the magnitude of the host is available, the second number (between parenthesis) is the number of $\mathrm{SN}$ for which the position of the $\mathrm{SN}$ relative to the center of the galaxy is also known. Bottom part: SN ratios computed from the first of the two numbers above, with statistical error. The data between parenthesis indicates what would be a $20 \%$ error resulting from missing systematically under-luminous $\mathrm{SN}$ from one of the sub-types; it is also the order of magnitude of the Shaw (1979) effect for $V_{\mathrm{HEL}}<2000 \mathrm{~km} \mathrm{~s}^{-1}$ (see text).

not be biased against the brighter ones. As long as the values of Richardson et al. (2002) (average and $1 \sigma$ standard deviation) do not cross the grey-shaded area, the majority of the SN should be present in the catalogue. Figure 1 thus shows that we may miss a significant number of events for SNII, SNIbc, and SNIa respectively beyond $V_{\mathrm{HEL}} \sim 5000,9000$, and $20000 \mathrm{~km} \mathrm{~s}^{-1}$. Thus, to avoid biasing our sample against or in favor of one of the SN subtypes, we have to cut it at $V_{\mathrm{HEL}}<5000 \mathrm{~km} \mathrm{~s}^{-1}$. Richardson et al. (2006) produced absolute magnitudes for Ib and Ic, of -17.98 (scatter of 0.46), and -18.14 (0.48) respectively for the so-called "normal" ones. These values are similar and slightly brighter than the "normal Ibc" taken from Richardson et al. (2002), so SNIb and SNIc should be biased neither against each other, nor against SNII adopting $V_{\mathrm{HEL}}<5000 \mathrm{~km} \mathrm{~s}^{-1}$. We note that here and in the rest of the paper, $\mathrm{SN}$ classified as $\mathrm{Ib} / \mathrm{c}$ are included when computing $N(\mathrm{Ibc})$, but are omitted when computing the ratio $N(\mathrm{Ib}) / N(\mathrm{Ic})$.

It can be seen in Fig. 1 that our SNIbc and SNIa are on average fainter than the absolute magnitude given by Richardson et al. (2002). This is probably because no extinction corrections are applied to the Asiago values, which also correspond to discovery magnitudes that may differ from peak magnitudes (the effect is less important for SN II-P, which are of about constant magnitude for a longer time than Ia or Ibc, making peak and discovery magnitudes closer to each other).

Because the Asiago catalogue is quite inhomogeneous and in view of the aforementioned magnitudes limits, we checked the robustness of our results by performing our analysis for samples defined in slightly different ways. In particular, we adopted a more conservative approach, defining a sample with $V_{\text {HEL }}<$ $2000 \mathrm{~km} \mathrm{~s}^{-1}$. This sample should be less affected by any residual bias but suffers from lower statistics. We also checked that older SN could be misclassified by performing our analysis when considering only events that occurred during and after the year 2000 with the disadvantage of a smaller number of events and larger error-bars. This affects mostly the trend found for $N(\mathrm{Ia}) / N(\mathrm{CC})$ as we discuss in Sects. 3.2, 4.1, and 4.2.

Richardson et al. (2002) note that about $20 \%$ of SN may be sub-luminous (with large uncertainties in this number). If the same fractions apply to all subtypes, our results, of course, would be unchanged. However, we should not consider that results are very robust whenever the obtained trends could be ascribed to a variation in the number of a subtype by this amount.

Another possible source of bias in SN host studies is the Shaw (1979) effect, i.e., the difficulty in detecting SN in the inner parts of distant galaxies, especially in photographic plates searches. This effect depends on the SN search programs (and is thus hard to quantify for the Asiago catalogue), although van den Bergh (1997) considers that it can be neglected for $\log \left(V_{\mathrm{HEL}}\right)<3.4$. Our conservative sample $\left(V_{\mathrm{HEL}}<2000 \mathrm{~km} \mathrm{~s}^{-1}\right)$ should thus be relatively unaffected. Cappellaro et al. (1993) state that within the same velocity limit $\left(V_{\mathrm{HEL}}<2000 \mathrm{~km} \mathrm{~s}^{-1}\right)$, $18 \%$ of all $\mathrm{SNe}$ are lost in the Asiago/Crimea searches in the overexposure of the central area of galaxies. If all of the SN lost are of a single type, the maximum error caused by this effect on a ratio is thus $18 \%$ in the $V_{\mathrm{HEL}}<2000 \mathrm{~km} \mathrm{~s}^{-1}$ sample. Cappellaro et al. (1993) measure $23 \%$ of lost SN for $V_{\mathrm{HEL}}<4000 \mathrm{~km} \mathrm{~s}^{-1}$ and $35 \%$ for their entire sample. These numbers are based on the $\mathrm{SN}$ missed in photographic searches, assuming CCD/Visual surveys do not miss any of the $\mathrm{SN}$ in the central regions. Thus, they could underestimate the true effect. However, we can assume that they provide an estimate of the uncertainty in the ratios (especially those derived from the $\mathrm{SN}$ radius) caused by the Shaw (1979) effect. A trend is considered robust if it creates a gradient larger than this uncertainty.

In the bottom panel of Fig. 1, we show the $N(\mathrm{Ibc}) / N(\mathrm{II})$, $N(\mathrm{Ib}) / N(\mathrm{Ic})$, and $N(\mathrm{Ia}) / N(\mathrm{CC})$ ratios within the volume defined by $V_{\mathrm{HEL}}$. The intersection with the vertical lines at 5000 (2000) $\mathrm{km} \mathrm{s}^{-1}$ corresponds to the number indicated in Table 1 for our adopted sample (and the "conservative" sample). We note that the $N(\mathrm{Ibc}) / N(\mathrm{II})$ and $N(\mathrm{Ib}) / N(\mathrm{Ic})$ ratios do not depend significantly on the velocity limit adopted. This confirms that we do not miss a large fraction of SNII versus SNIbc (and SNIb versus SNIc) within our samples ${ }^{2}$; this may not be so surprising as the average absolute magnitudes differ by relatively small amounts of $-17.23 \pm 0.62$ versus $-16.61 \pm 1.23$, and $-17.98 \pm 0.46$ versus $-18.14 \pm 0.48$. The ratios $N(\mathrm{Ib}) / N(\mathrm{Ic})$, and $N(\mathrm{Ibc}) / N(\mathrm{II})$ are thus quite robust, at least in the local universe ${ }^{3}$. In contrast, the $N(\mathrm{Ia}) / N(\mathrm{CC})$ ratio increases continuously when including more distant SN (i.e., going from $V_{\mathrm{HEL}}<2000 \mathrm{~km} \mathrm{~s}^{-1}$ to $V_{\mathrm{HEL}}<$ $5000 \mathrm{~km} \mathrm{~s}^{-1}$ ). Although the limitation $V_{\mathrm{HEL}}<5000 \mathrm{~km} \mathrm{~s}^{-1}$ should still allow us to derive meaningful results, we note that this ratio does depend on the limit adopted and should thus be considered relatively uncertain.

Adopting $V_{\mathrm{HEL}}<5000 \mathrm{~km} \mathrm{~s}^{-1}$, the catalogue provides 1038 $\mathrm{SN}$, of which 701 occur in star forming galaxies (spirals and irregulars) with at least the host galaxy magnitude known. The sample that we use to study the core-collapse ratios is then a factor 2.5 larger than the $280 \mathrm{CCSN}$ used in PB03. The distribution of SN types in this sample is given in Table 1.

The $\mathrm{SN}$ ratios that we obtain for the different $\mathrm{SN}$ types of our sample are consistent with published values: the ratio of SNIbc to SNII supernovae in our sample is $N(\mathrm{Ibc}) / N(\mathrm{II})=0.31 \pm 0.04$, similar to 0.27 in PB03; $0.23 \pm 0.05$ in Bressan et al. (2002); 0.33 in Hamuy (2002); 0.41 in Smartt et al. (2009); and 0.3, 0.16 , and 0.31 for respectively $\mathrm{S} 0 \mathrm{a} / \mathrm{b}, \mathrm{Sbc} / \mathrm{d}$ and Irr galaxies in Mannucci et al. (2005). The ratio of thermonuclear to core collapse $\mathrm{SN}$ in that sample is $N(\mathrm{Ia}) / N(\mathrm{CC})=0.4 \pm 0.04$, and the

\footnotetext{
2 In the worst case scenario, it would mean that we miss similar fractions of SNII versus SNIbc (and SNIb versus SNIc) when we push the velocity limit to higher values.

3 Since the $\mathrm{N}(\mathrm{Ibc}) / \mathrm{N}$ (II) ratio depends on metallicity (PB03) and since cosmic metallicity decreases, on average, with redshift, that ratio is expected to decline with redshift, albeit very slowly.
} 
same ratio is obtained from the local universe supernovae sample (Smartt et al. 2009). Using the rates from Mannucci et al. (2005), we obtain for that ratio the values $0.41,0.19$, and 0.34 in $\mathrm{S} 0 \mathrm{a} / \mathrm{b}, \mathrm{Sbc} / \mathrm{d}$, and Irr galaxies, respectively; the corresponding uncertainties, however, are very large, due to small statistics. Our result for $N(\mathrm{Ic}) / N(\mathrm{Ib})=1.65 \pm 0.32$ is consistent with the one obtained from the local universe sample of Smartt et al. (2009): $N(\mathrm{Ic}) / N(\mathrm{Ib})=2$ considering the small statistics $(27 \mathrm{SN})$ in their sample for this ratio (compensated however by a careful checking of the data for every SN used in their work).

\section{Dependence of $\mathrm{SN}$ type ratios on global galaxy properties}

Using a sample of $280 \mathrm{CCSN}$ from an earlier version of the Asiago catalogue, PB03 found that the $N(\mathrm{Ibc}) / N(\mathrm{II})$ ratio has an average value of $\sim 0.30$, whereas it increases with host galaxy magnitude. They interpreted the latter as an effect of the global galaxian metallicity (increasing with galaxian luminosity) on the masses of the precursors of the CCSN sub-types: as metallicity increases, the stellar enveloppe is more easily lost and lower mass stars may become SNIbc, increasing thus the $N(\mathrm{Ibc}) / N(\mathrm{II})$ ratio. Assuming that all CCSN are produced by single stars, and that the physical reason for a star to explode as a SNIc, SNIb, or SNII is only its initial mass (see the discussion in Sect. 5 for other possibilities), the $N(\mathrm{Ibc}) / N(\mathrm{II})$ ratio is expressed as

$\frac{N(\mathrm{Ibc})}{N(\mathrm{II})}=\frac{\int_{M_{\mathrm{Ibc}}}^{M_{\mathrm{Up}}} \Psi\left(t-\tau_{M}\right) \Phi(M) \mathrm{d} M}{\int_{M_{\mathrm{II}}}^{M_{\mathrm{lbc}}} \Psi\left(t-\tau_{M}\right) \Phi(M) \mathrm{d} M}$

where $\Psi(t)$ is the star formation rate at time $t, \tau_{M}$ is the lifetime of star of mass $M, \Phi(M)$ is the stellar initial mass function (IMF), and $M_{\mathrm{Up}}, M_{\mathrm{II}}$, and $M_{\mathrm{Ibc}}$ are respectively, the upper mass limit of the IMF (around $100 M_{\odot}$ ), the lower mass limit for a star to explode as a SNII (around $8 M_{\odot}$, see e.g. Smartt et al. 2009; Anderson \& James 2008), and the lower mass limit for a star to explode as a SNIbc. Based on the crucial assumption of stationarity, whereby the progenitor lifetimes of both classes of SN (i.e., SNII and SNIbc) are short compared to the duration of the corresponding star formation episodes, $\Psi$ can be taken from the integrals in Eq. (1) (since the progenitors of both classes of SN have similar lifetimes, during which $\Psi$ varies very little). Then, Eq. (1) reads

$$
\frac{N(\mathrm{Ibc})}{N(\mathrm{II})}=\frac{\int_{M_{\mathrm{Ibc}}}^{M_{\mathrm{Up}}} \Phi(M) \mathrm{d} M}{\int_{M_{\mathrm{II}}}^{M_{\mathrm{Ibc}}} \Phi(M) \mathrm{d} M}
$$

i.e., the $N(\mathrm{Ibc}) / N(\mathrm{II})$ ratio is a function of the slope of the IMF and of $M_{\mathrm{Ibc}}$. For a given IMF, if $M_{\mathrm{Ibc}}$ increases with metallicity, the ratio given by Eq. (2) will obviously decrease and the same is also true if one replaces $N(\mathrm{Ibc}) / N(\mathrm{II})$ by $N(\mathrm{Ic}) / N(\mathrm{Ib})$ and $M_{\mathrm{Ibc}}$ by $M_{\mathrm{Ic}}$. We note that Anderson \& James (2008) suggested such a hierarchy of limiting masses between SNII, SNIb, and SNIc on the basis of the association of SN with regions of recent star formation traced by $\mathrm{H} \alpha$ emission. Their findings indicate that $M_{\mathrm{II}} \sim 7.8 M_{\odot}$. Kelly et al. (2008) found that SNIc occur in the brightest regions of their host, where the most massive stars probably form, also suggesting that SNIc result from the explosion of the most massive stars.

\subsection{The $N(I b c) / N(I I)$ and $N(I c) / N(I b)$ ratios}

We first repeat the analysis as PB03 for the ratios of CCSN subtypes with our larger sample. In Fig. 2, the top panels show the $N(\mathrm{Ibc}) / N(\mathrm{II})$ ratio versus $M_{B}$. Four bins in $M_{B}$ with $\sim 100 \mathrm{CCSN}$ in each one are constructed and the corresponding $M_{B}$ value is taken as the median value of $M_{B}$ in each bin. In the insert panel, it is evident that in bins of constant CCSN numbers, $N(\mathrm{Ibc})$ increases for brighter galaxies (and $N(I I)$ decreases by the same amount), causing the $N(\mathrm{Ibc}) / N(\mathrm{II})$ ratio to increase with galaxian luminosity. The resulting $N(\mathrm{Ibc}) / N(\mathrm{II})$ versus $M_{B}$ relation is quite similar to the one obtained in PB03 (dotted curve), with smaller vertical error bars, reflecting the larger size of the new sample. We thus confirm the detection of PB03 of a correlation between the $N(\mathrm{Ibc}) / N(\mathrm{II})$ ratio and global galaxian metallicity, which is also supported by the study of Prieto et al. (2008). The only limitation of this conclusion is obtained with the conservative sample for which the relation is rather flat. The main difference come from the bin corresponding to the brightest galaxies showing low values with respect to the $V_{\mathrm{HEL}}<5000 \mathrm{~km} \mathrm{~s}^{-1}$ sample and the PB03 fit (error bars are however rather large). We return to the interpretation of that result in Sect. 5, after presenting in Sect. 4 the results of our study concerning that same ratio as a function of local metallicity.

The $N(\mathrm{Ic}) / N(\mathrm{Ib})$ ratio (middle panel of Fig. 2) has considerably larger uncertainties than the $N(\mathrm{Ibc}) / N(\mathrm{II})$ ratio, because of poorer number statistics. Indeed, each one of the four bins contains $\sim 8 \mathrm{SNIb}$ and $\sim 12$ Ic (insert in middle panel), for an average ratio of $N(\mathrm{Ic}) / N(\mathrm{Ib}) \sim 1.6$ That ratio shows no clear variation with $M_{B}$. At first sight, this appears to indicate an opposite situation to the case of the $N(\mathrm{Ibc}) / N(\mathrm{II})$ ratio. We shall see, however, in Sect. 4 , that the situation is different when $N(\mathrm{Ic}) / N(\mathrm{Ib})$ is expressed as a function of local metallicity. This "puzzling" behaviour is discussed further in Sect. 4 and attributed to small number statistics.

\subsection{The $N($ la $) / N(C C)$ ratio}

We extend our study to the $N(\mathrm{Ia}) / N(\mathrm{CC})$ ratio as a function of host galaxy $M_{B}$. As can be seen in the bottom panels of Fig. 2, there is also a clear trend in that case, such that brighter galaxies hosts proportionally more SNIa than CCSN. To our knowledge, this is the first time that this correlation has been detected. In view of the numbers of SN involved (see insert panel), we propose that this result is robust, in contrast to the case for $N(\mathrm{Ic}) / N(\mathrm{Ib})$. We note that we identify this correlation for the conservative limit $V_{\mathrm{HEL}}<2000 \mathrm{~km} \mathrm{~s}^{-1}$ (right panel), although its slope decreases in that case. On the other hand, taking only recent $\mathrm{SN}$ from the sample (trying to avoid misclassifications), the correlation becomes steeper. The dependence of $N(\mathrm{Ia}) / N(\mathrm{CC})$ on the magnitude is thus relatively uncertain in terms of absolute value; however since we do find a correlation with magnitude in every one of our tests, we conclude that it is real.

This result can be translated in terms of metallicity, larger $N(\mathrm{Ia}) / N(\mathrm{CC})$ ratios being found in more metal-rich galaxies. We see indeed in Sect. 4 that this result also holds when the local metallicity is considered. However, we understand that, in contrast to the previous case, there is no causal relationship here, i.e., metallicity does not in any way affect the $N(\mathrm{Ia}) / N(\mathrm{CC})$ ratio. Instead, it is the mass of the host galaxy that affects this ratio, in a way that can most easily be seen as follows.

The CCSN rate of a galaxy is proportional to the star formation rate $\Psi$

$N(\mathrm{CC}) \propto \Psi$. 


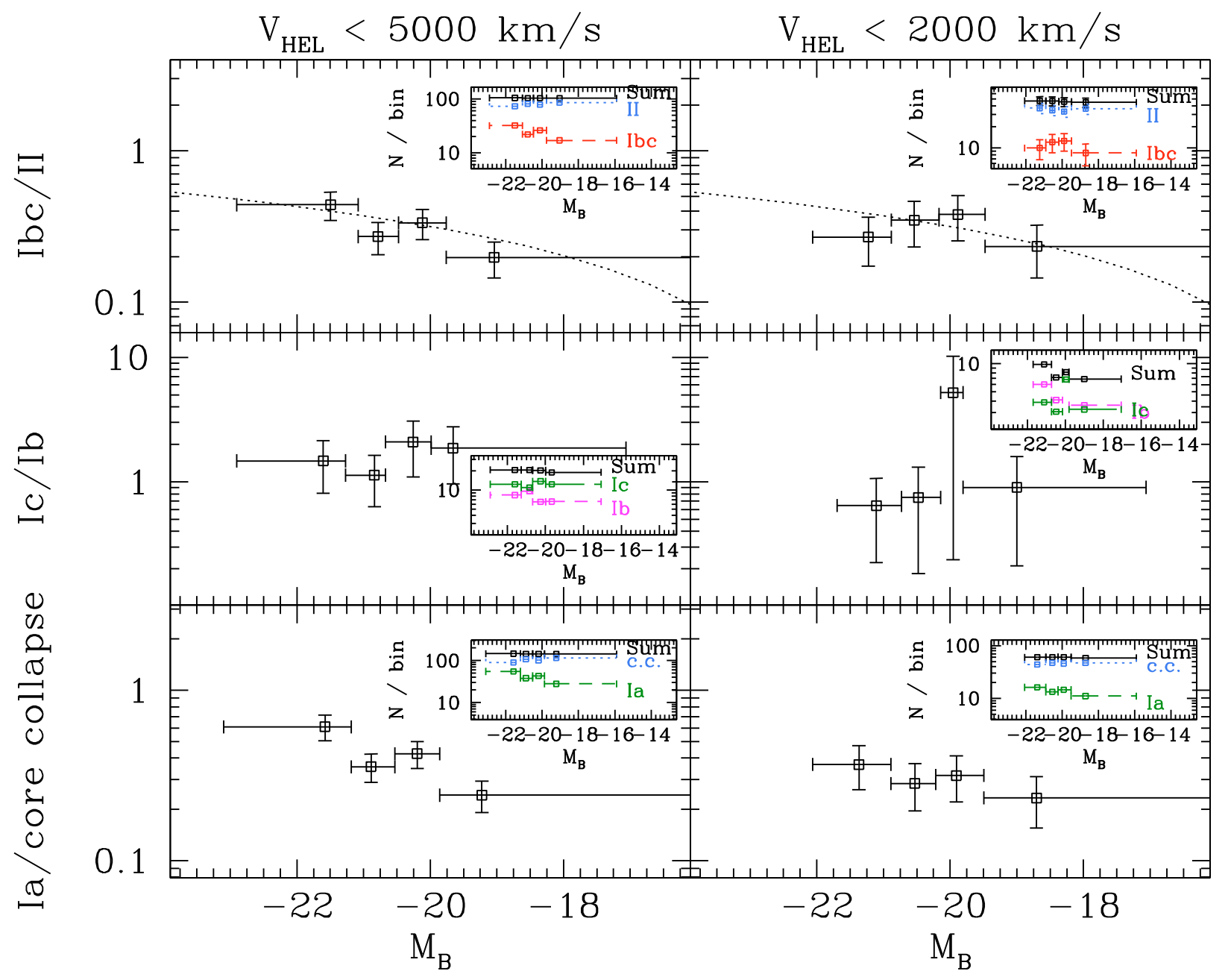

Fig. 2. From top to bottom: number ratios of $N(\mathrm{Ibc}) / N(\mathrm{II}), N(\mathrm{Ic}) / N(\mathrm{Ib})$, and $N(\mathrm{Ia}) / N(\mathrm{CC})$, as function of galaxian blue magnitude $M_{B}$ for the conservative $V_{\mathrm{HEL}}<2000 \mathrm{~km} \mathrm{~s}^{-1}$ sample (right) and the $V_{\mathrm{HEL}}<5000 \mathrm{~km} \mathrm{~s}^{-1}$ sample (left, for more robust statistics). The inserts in each panel display the corresponding numbers of each SN type and are chosen such that each $M_{B}$ bin has the same total number of SN of the types involved. The dotted curve in the upper panel is the fit to the data of PB03, which had much larger error bars.

The SNIa rate is more difficult to evaluate, since thermonuclear supernovae may result from stars of all ages, not just the young ones. Scannapieco \& Bildsten (2005) introduced a useful parametrization, describing the SNIa rate as a sum of two terms, one depending on the current star formation rate and the other on the total stellar mass $M_{*}$ (i.e., the time integrated star formation rate). That parametrization (adopted by e.g., Sullivan et al. 2006; Aubourg et al. 2008) reproduces most available data satisfactorily and we adopt it here

$N(\mathrm{Ia})=\alpha \Psi+\beta M_{*}$,

where $\alpha$ and $\beta$ are constants. Thus, the required ratio can be written as

$\frac{N(\mathrm{Ia})}{N(\mathrm{CC})}=A+B \frac{M_{*}}{\Psi}$.

It is well known that larger galaxies have, on average, lower specific star formation rates $\Psi / M_{*}$ because of their smaller gas fractions (e.g., Boissier et al. 2001; Boissier \& Prantzos 2000, and references therein), i.e., the ratio $M_{*} / \Psi$ is, on average, an increasing function of galaxy mass and luminosity. Thus, both the metallicity of a galaxy and its $N(\mathrm{Ia}) / N(\mathrm{CC})$ ratio increase with its mass. This explains, at least qualitatively, the correlation found in the bottom panels of Fig. 2.

\section{Dependence of SN type ratios on local galaxy properties}

PB03 used the absolute magnitude of the host galaxy as a proxy for its global metallicity, based on the well established magnitude-metallicity relationship. However, disk galaxies are known to exhibit metallicity gradients (e.g., Henry \& Worthey 1999; Zaritsky et al. 1994; van Zee et al. 1998). If metallicity indeed affects the ratios of SN types, a radial effect should also be found. Hakobyan (2008) demonstrated that the radial distributions of SNIbc and SNII differ, more SNIbc being found at smaller radii than SNII (also using a sample extracted from the Asiago catalogue, but with a different selection from ours), A similar analysis was completed by van den Bergh (1997), for a smaller sample of $156 \mathrm{SN}$.

\subsection{Dependence on galactocentric distance}

We investigate whether this radial effect is seen in our sample, using the supernovae for which we can compute a galactocentric radius. This is possible when the catalogue provides the offset of the SN, the position angle, and inclination of the host galaxy, as well as its $R_{25}$ radius, which is needed to normalize the results. From these parameters, we can compute the distance between 


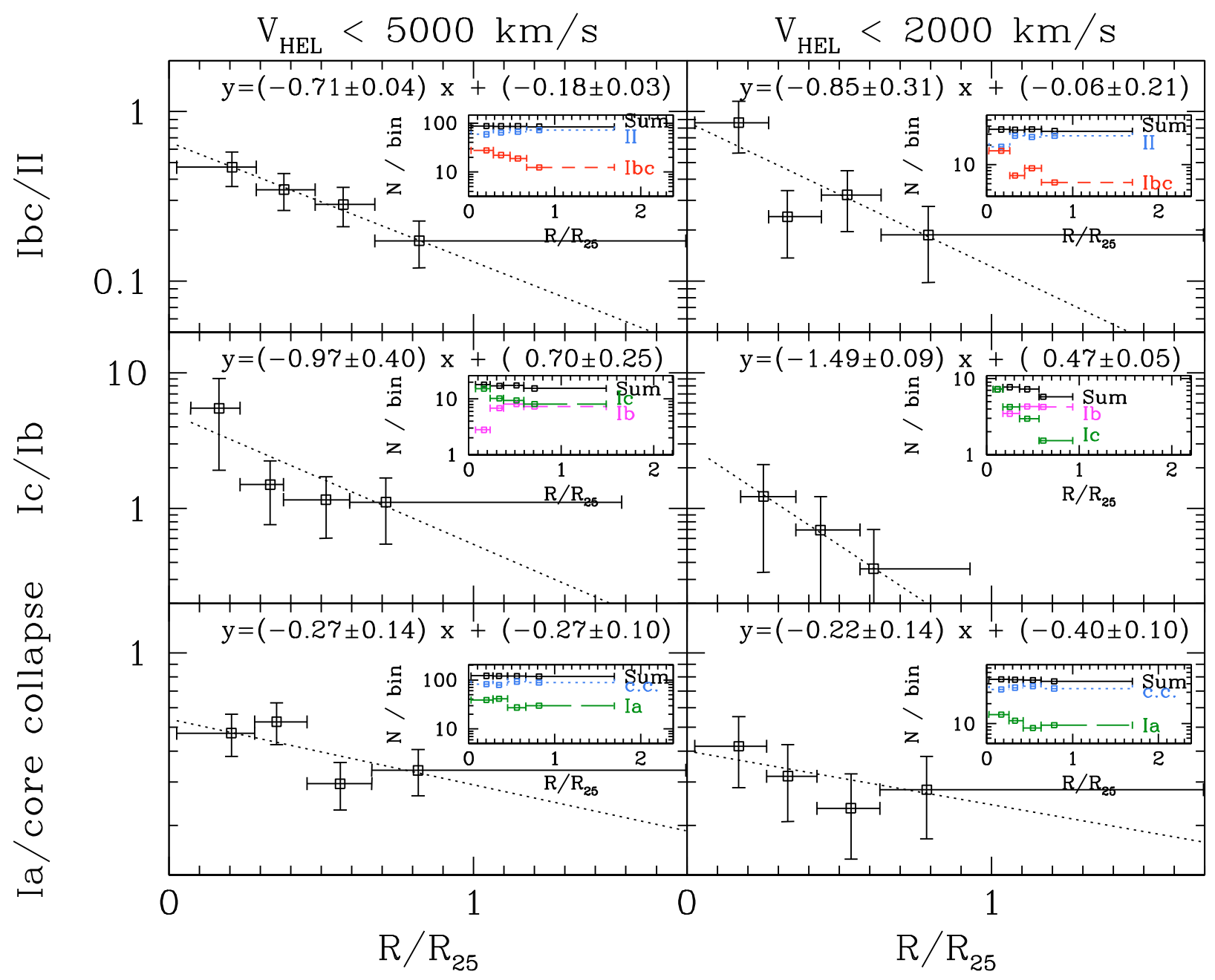

Fig. 3. Same thing as in Fig. 2, but this time as a function of galactocentric radius, expressed in units of $R_{25}$.

the SN position and the center of the galaxy, within its plane, that we refer to as the galactocentric radius of the SN. We note that we deproject the minor axis by simply dividing its value by $b / a=\cos ($ inclination angle), and we do not consider galaxies that are almost edge-on (inclination larger than 80 degrees). These restrictions reduce the size of the usable sample for this part of the study, but only moderately (see Table 1) allowing us to work with reliable number statistics.

Figure 3 displays the same ratios as Fig. 2, this time as a function of galactocentric radius. In the case of $N(\mathrm{Ibc}) / N(\mathrm{II})$, a clear trend is observed. SNIbc are found at smaller normalised radius than SNII, in agreement with Hakobyan (2008). van den Bergh (1997) suggested from his small sample that SN Ibc are more centrally concentrated in their host galaxy than SNII. As mentioned in Sect. 2, the Shaw (1979) effect should be lower than $35 \%$, but the difference between the inner and outer bins is much larger (about a factor 2.5). Adopting the conservative sample (right panel), the Shaw (1979) effect should be even smaller, and we still find a trend (which is even stronger: slope of -0.85 instead of -0.71 ). Thus, we believe that this trend is unaffected by this source of bias. The easiest way to interpret this observation is in term of metallicities: higher metallicities are found in the inner parts of galaxies, leading to a lower limiting mass for type Ibc supernovae. We quantify this effect in Sect. 5.2 in terms of local metallicities, showing that it is consistent with what we obtained in Sect. 4 by using global metallicities.
The clear trend obtained in the case of $N(\mathrm{Ic}) / N(\mathrm{Ib})$ (middle panels in Fig. 3) is rather surprising, in view of the results of Sect. 2: $N(\mathrm{Ic}) / N(\mathrm{Ib})$ apparently increases with decreasing galactocentric radius (i.e., with increasing metallicity), while no variation with $M_{B}$ is seen in Fig. 2. We note that with a difference between inner and outer bins of a factor about 5, here again, we cannot attribute the observed trend to the Shaw (1979) effect (if we adopt the conservative sample, we do not have any SN $\mathrm{Ib}$ in the innermost bin, but the trend in the three other bins is stronger). We attribute this striking difference in the trend with magnitude and radius to the small number statistics involved in the evaluation of that ratio. As emphasized at in the beginning of Sect. 4, the assumption of stationarity is crucial to the evaluation of the various SN ratios, and is naturally fullfilled if large numbers of $\mathrm{SN}$ are involved. In this case, the formation times of the SN progenitors of all types span the entire range of the progenitor lifetimes $\left(\tau_{M}\right)$; an average $\Psi$ can then be used, allowing one to pass from Eq. (1) to Eq. (2). However, in the case of small numbers of SN the situation is different: if a few starbursts occurred recently (less than a few Myr ago), only the most massive of their stars have had time to explode up to now, favouring SNIc (presumably resulting from more massive stars) over SNIb (and, for the same reasons, SNIbc over SNII). In that case, the term $\Psi\left(t-\tau_{M}\right)$ in Eq. (1) does not cancel out with the corresponding term in the denominator and may mask the effect of any metallicity dependence of $M_{\mathrm{Ibc}}$ (the dividing mass between 
SN exploding as Ibc or II) or of $M_{\text {Ic }}$ (the dividing mass between $\mathrm{SN}$ exploding as Ib or Ic). We shall see in the next section that the radial trend of $N(\mathrm{Ic}) / N(\mathrm{Ib})$ found here translates directly into a local metallicity trend, but because of low number statistics it is impossible to draw meaningful conclusions.

Finally, the bottom panels of Fig. 3 displays the ratio of thermonuclear to core collapse supernovae $N(\mathrm{Ia}) / N(\mathrm{CC})$. The ratio appears to increase in the inner galaxian zones. The trend is relatively weak: for the slope and uncertainty in the figure, a Student's t-test indicates a $\sim 20 \%$ probability of the null hypothesis that there is no dependence of $N(\mathrm{Ia}) / N(\mathrm{CC})$ on the radius. Similar results are obtained for the conservative sample $\left(V_{\mathrm{C}}<2000 \mathrm{~km} \mathrm{~s}^{-1}\right)$. Another worrying issue is that inner and outer bins are different only by a factor $\sim 1.4$. This is still larger than the typical uncertainties due to the Shaw (1979) effect or underluminous SN, but, in combination with the large statistical error bars, it makes this trend less robust in terms of the uncertainties than the other ones presented in the figure. Interestingly, if we keep only SN that exploded during or after the year 2000, avoiding possible misclassifications for older SN, we find a steeper trend with radius (slope $-0.46 \pm 0.06$, reducing the probability for the null hypothesis to less than 1\%). In summary, although the trend in Fig. 3 is not very strong, it is reasonable to believe that it is real because it persists when the size sample is reduced by various criteria in attempting to improve the quality of our results. It is reassuring that this trend is also expected on the basis of the analysis made in Sect. 3.2. Indeed, the gaseous profiles of disk galaxies vary little with galactocentric radius, while the stellar ones vary much more (for instance, in the case of the Milky Way disk the scalelength of the stellar profile is $\sim 2.5 \mathrm{kpc}$, while the one of the gas $\sim 8 \mathrm{kpc}$; see e.g., Boissier \& Prantzos (1999) for observed stellar and gaseous profiles of the MW disk). This implies that the term $M_{*} / \Psi$ in Eq. (5) is expected to increase in the inner galaxian zones and so will do the corresponding $N(\mathrm{Ia}) / N(\mathrm{CC})$ ratio. We develop this argument further analytically in Sect. 5.3 and illustrate it with a numerical example for the case of the Milky Way disk. We shall see in the next section that this radial trend is also expressed in terms of local metallicity; however, as already argued in Sect. 2, metallicity is not the root cause of that effect.

\subsection{Dependence on local metallicity}

To probe the metallicity dependence of SN types, one may use direct or indirect methods to determine metallicity. Direct measurements of abundances of host galaxies of supernovae are possible only for moderately large samples of SN (e.g., 254 galaxies from the SDSS in Prieto et al. 2008) and these are integrated abundances over the entire galaxy, not at the supernova position. The Measurement of local metallicities, i.e., in HII regions at the immediate vicinity of the supernova, would be ideal but it would require an enormous observational effort before reasonable statistics can be obtained. Determinations of local metallicities from spectroscopy of neighboring HII regions do exist (Smartt et al. 2009; Modjaz et al. 2008) but only for a relatively small number of events, not allowing the statistical study of the various trends explored in this work.

In PB03, we used an indirect way, i.e., the well-known massmetallicity relationship to evaluate the metallicity of the host galaxies (of late type) of CCSN. In this work, we use known relationships among disk galaxies to derive again in an indirect (and approximate) way the local metallicity of galaxies at the galactocentric radius of the supernovae of our sample.

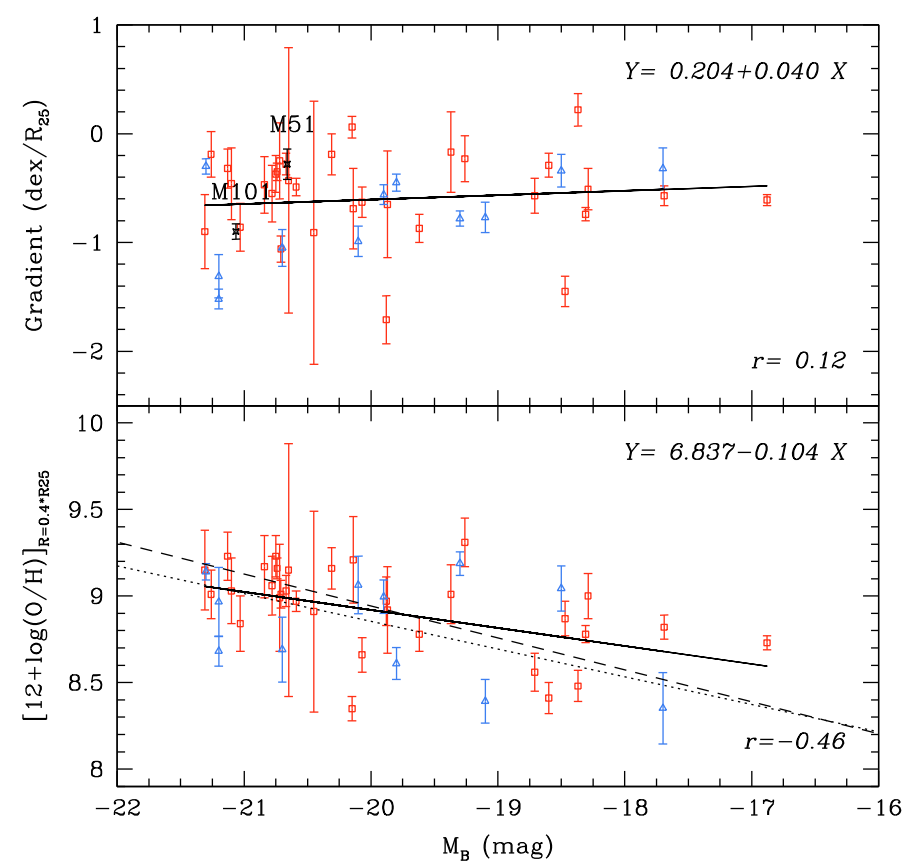

Fig. 4. Top: metallicity (oxygen) gradient per $R_{25}$ as a function of absolute magnitude $M_{B}$. Bottom: characteristic metallicity (at $0.4 R_{25}$ ) as a function of $M_{B}$. Data in both panels are from Zaritsky et al. (1994) (squares) and van Zee et al. (1998) (triangles). $r$ is the correlation coefficient, and the solid line indicates in each case the best linear least squares fit (the parameters of the line are given in each panel). In the top panel, the gradients derived by Bresolin et al. (2004) and Bresolin (2007) for M 51 and M 101 are overplotted. In the bottom panel, dashed and dotted lines indicate, respectively the empirical relations obtained by Tremonti et al. (2004) and Garnett (2002) using respectively global metallicities (measured on the integrated galaxy) and metallicities at the effective (half-light) radius. The latter was used as a proxy for the global metallicity in PB03. We apply the same approach in this paper for the global metallicity.

It is known that the abundance gradient in nearby disk galaxies has a universal value when expressed in dex $/ R_{25}$ (e.g., Henry $\&$ Worthey 1999) of $\mathrm{d} \log (\mathrm{O} / \mathrm{H}) / \mathrm{d} R_{25} \sim-0.6 \mathrm{dex} / R_{25}$. With detailed semi-analytical models of disk galaxy evolution, Prantzos $\&$ Boissier (2000) showed that this universality can indeed be reproduced, thus confirming an earlier suggestion of Garnett et al. (1997) about "homologuous" disk evolution. Combining this empirical fact with the observed luminosity-metallicity relation, it is possible to deduce the metallicity at the vicinity of the $\mathrm{SN}$ from the luminosity of the host galaxy and the galactocentric radius of the $\mathrm{SN}$, which was evaluated in Sect. 4.1.

For our purpose, we use the data of two studies of abundance gradients with relatively large samples of nearby galaxies: Zaritsky et al. (1994) and van Zee et al. (1998). We show in Fig. 4 (top) the abundance gradients in $\operatorname{dex} / R_{25}$, which display little variation (if any at all) with absolute magnitude. In the bottom panel of Fig. 4, we display the abundance measured at $0.4 R_{25}$ as a function of the absolute $B$ band magnitude $M_{B}$ (the metallicityluminosity relation). Each panel features the linear least squares fit to these relationships (solid lines) that we adopt in this work to compute the local metallicity. The dashed and dotted lines in the bottom panel of Fig. 4 indicate the metallicity-luminosity relations of Tremonti et al. (2004) and Garnett (2002). The first one was obtained from integrated galaxy spectra of the Sloan Digital Sky Survey. The second one displays the metallicity at the effective (half-light) radius and was used in PB03 to estimate the 


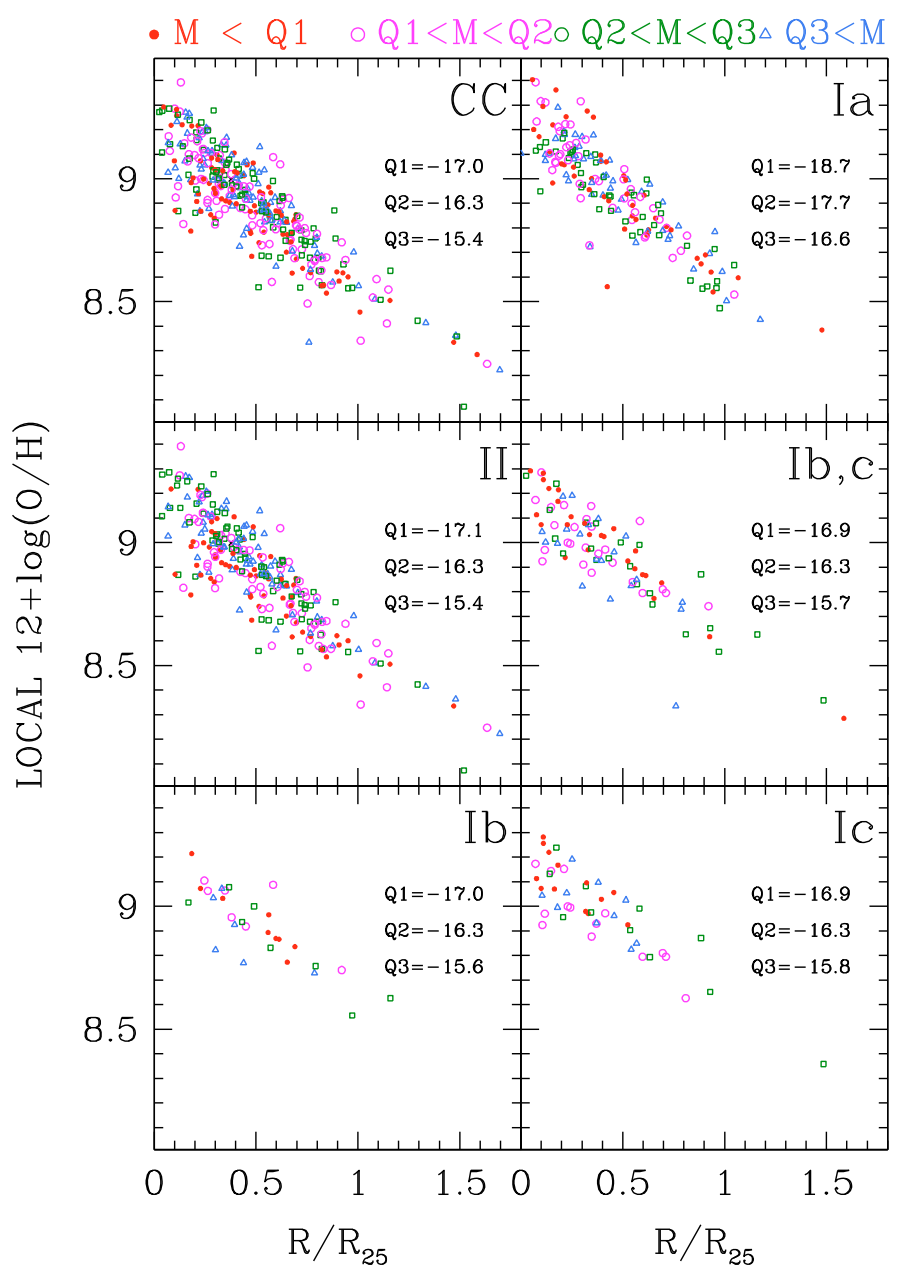

Fig. 5. Metallicities of the various $\mathrm{SN}$ types of our sample, as a function of their position (expressed in $R / R_{25}$ ) inside their host galaxy. Metallicities are derived from the $\mathrm{SN}$ position in and the magnitude of the host galaxy, according to the procedure described in Sect. 5.2 and Eq. (6). The color/type coding is completed according to the absolute magnitude of the SN with respect to the three quartiles Q1, Q2, and Q3 indicated in each panel.

metallicity in SN hosts without knowing the galactocentric distance of the SN. In this paper, for consistency, we still use it to estimate the global metallicity. Despite using different definitions of the "characterisitic" abundance, the aforementioned relationships are very close to each other, and our results would be marginally affected by the use of either of them.

Assuming that the two empirical relations (i.e., the solid lines in the top and bottom panels) are valid for all galaxies in our sample, we can compute the metallicity profile (in terms of $R / R_{25}$ ) of each galaxy from its luminosity. We can then easily evaluate the local metallicity at the galactocentric radius of the supernova. Using the numerical values of Fig. 4 (i.e the fits appearing in each panel), we obtain

$$
\begin{aligned}
{[12+\log (\mathrm{O} / \mathrm{H})](R)=} & 6.837-0.104 M_{B} \\
& +\left(\frac{R}{R_{25}}-0.4\right)\left(0.204+0.04 M_{B}\right) .
\end{aligned}
$$

We note that, although the abundance gradient has an almost universal value in dex $/ R_{25}$, our fit indicates a slight trend with $M_{B}$, which is expressed by the last term (dependence on $M_{B}$ ) in the derived expression. We retain this term for consistency, but it is clear that, in view of its small magnitude, it has no influence on the results.

The resulting local metallicities $\mathrm{O} / \mathrm{H}(R)$ as a function of normalized galactocentric radius $R / R_{25}$ are given in Fig. 5 for all the SN of our sample: the upper panels displays the results for CCSN and SNIa, the middle panels SNII and SNIbc, and the bottom panels SNIb and SNIc. In all cases, the metallicity gradient is the same, but the absolute value of the metallicity at each normalized radius depends on the corresponding host galaxy magnitude. It is the first time that this technique has been used to derive the local metallicities of SN progenitors. These results obviously depend on how accurately the adopted average relationships (metallicity-luminosity and gradient-luminosity) apply to each galaxy of our sample.

After deriving the metallicities at the position of each supernovae in this way, we then compute the corresponding ratios as a function of the local metallicity. In Figs. 6 and 7, we present the results for the ratios $N(\mathrm{Ibc}) / N(\mathrm{II})$ and $N(\mathrm{Ic}) / N(\mathrm{Ib})$ respectively as a function of global metallicity (top, after the results of Sect. 4.1 and the magnitude-metallicity relationship) and of local metallicity (bottom).

Before discussing these results, we note that we created the same figure adopting different abundance gradients. In the past few years, several studies have mentioned the possible errors in abundances obtained from strong lines as in Zaritsky et al. (1994) or van Zee et al. (1998). Bresolin et al. (2004) and Bresolin (2007) performed a more detailed analysis of the gradients in M 51 and M 101. They found flatter gradients than in previous studies for the same galaxies, although their gradients are within the dispersion of those in Fig. 4. We performed the same analysis as described above but adopting the two values for M 51 and M 101 rather than our fit, but found that our results were unchanged qualitatively. The only difference is that flatter gradients cause the trend with metallicity to become steeper and vice versa.

In the case of $N(\mathrm{Ibc}) / N(\mathrm{II})$, the results of the $V_{\mathrm{HEL}}<$ $5000 \mathrm{~km} \mathrm{~s}^{-1}$ as a function of global metallicity are consistent with those obtained in both PB03 (dotted curve) and Prieto et al. (2008, vertical dotted error bars). We note the relatively good statistics, due to the size of our sample (see insert). The corresponding ratios as a function of local metallicity (bottom panel) are quite consistent with those obtained for global metallicity and the statistics is almost equivalent. Looking at the $V_{\mathrm{HEL}}<$ $2000 \mathrm{~km} \mathrm{~s}^{-1}$ sample, we note that the trend with global metallicity is consistent with the absence of relationship. However, this is strongly dependent on the higher metallicity bin (the three other data points remain indeed within $1 \sigma$ of the PB03 fit). We think that low number statistics are partly responsible for this difference. The relation with local metallicity however (which should be a more reliable estimator of the true progenitor metallicity) is totally consistent with the one obtained with the $V_{\mathrm{HEL}}<5000 \mathrm{~km} \mathrm{~s}^{-1}$ sample. We conclude that the trend between $N(\mathrm{Ibc}) / N(\mathrm{II})$ and metallicity, first identified in PB03, is firmly established as long as the two types Ibc and II are unbiased as a function of $R / R_{25}$. The discussion in Sect. 4.1 suggests that this is the case. In Fig. 5, the symbol/color coding according to the $\mathrm{SN}$ magnitudes also shows that $\mathrm{SN}$ in the inner galactic regions are apparently not biased towards the brighter ones. The case of $N(\mathrm{Ic}) / N(\mathrm{Ib})$ is much less clear. Results show no trend with global metallicity (as expected from Sect. 3.1) but they do show a trend as a function of local metallicity (as expected from Sect. 4.1), even if the slope and its intersect are poorly constrained (see the error bars and the difference between the two samples shown in Fig. 7). Because of the smaller samples (see inserts), 


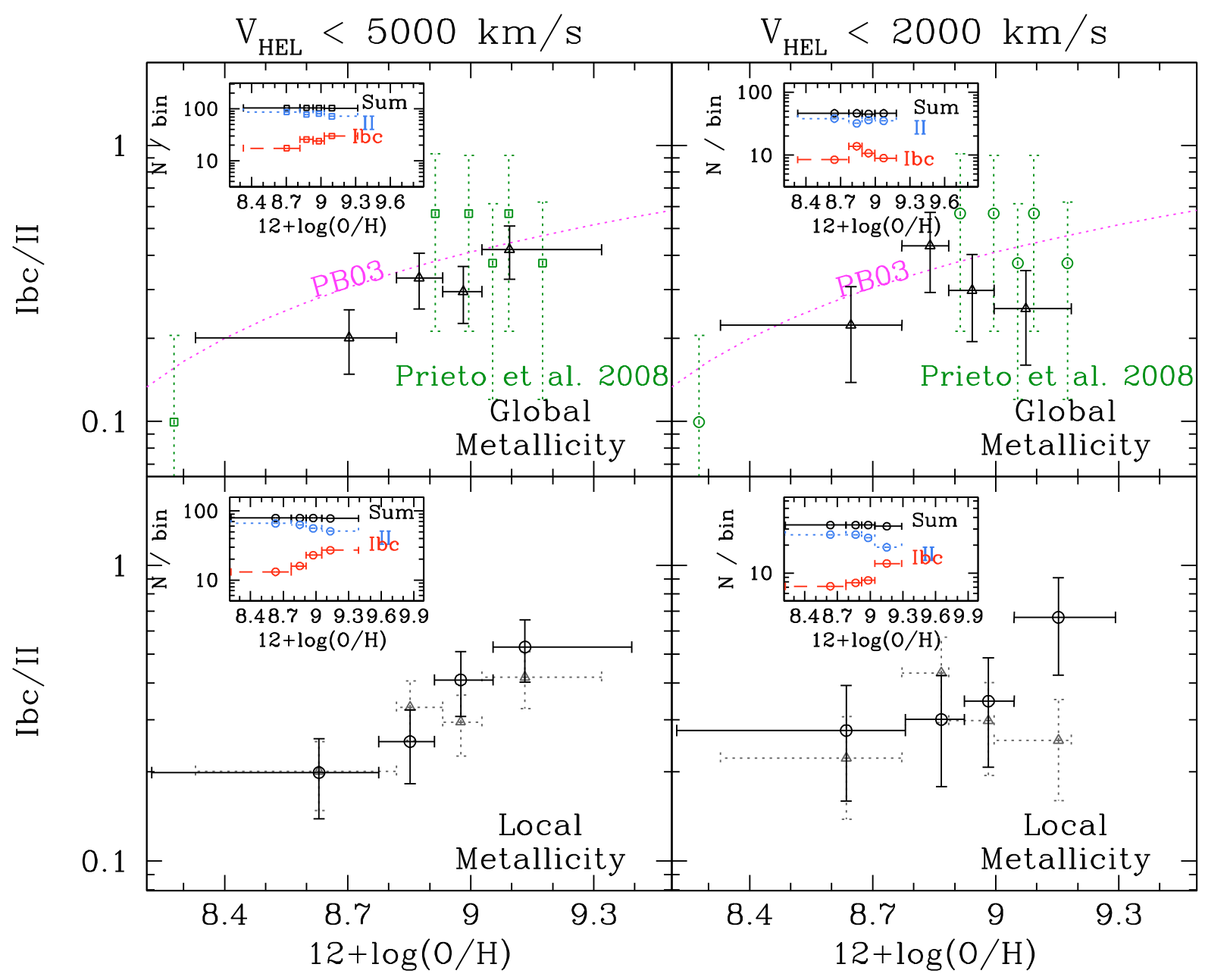

Fig. 6. Symbols with solid errror bars: number ratios of SNIbc/SNII as a function of global galaxian metallicity (top, obtained from the magnitudemetallicity relation of Fig. 4) and of local metallicity (bottom, obtained from Eq. (6)), for the conservative $V_{\mathrm{HEL}}<2000 \mathrm{~km} \mathrm{~s}^{-1}$ sample (right) and the $V_{\mathrm{HEL}}<5000 \mathrm{~km} \mathrm{~s}^{-1}$ sample (left, better statistics). Inserts in all panels display corresponding numbers of SN in each bin (chosen such as the total numbers are approximately equal). Triangles with dotted error bars in lower panels are the same as those with solid error bars in the upper panels and are displayed for comparison with local metallicity results. In the upper panels, the dotted curve is the fit to the data of PB03 and the symbols with dotted vertical error bars the data collected in Prieto et al. (2008).

statistics is poorer in that case, as reflected in the large error bars. The arguments developped in Sect. 4.1 suggest that such a conflicting situation can indeed occur: Eq. (2) does not apply and Eq. (1) (which always applies) can produce ambiguous results, depending on the ages of the few starbursts involved. It is then impossible to draw any conclusions about the dependence of this ratio on metallicity; a substantially larger sample is required for that.

Finally, our results for the SNIa/CCSN ratio are plotted in Fig. 8 as a function of global (top) and local (bottom) metallicities. Results are plotted for the two samples with values of the maximal heliocentric velocities of the host galaxies $V_{\mathrm{HEL}}<$ 2000 , and $5000 \mathrm{~km} \mathrm{~s}^{-1}$. The left and right panels show the results using respectively all the $\mathrm{SN}$ and only the recent ones. It is clearly seen that:

i) For global metallicities, the slope of the relation differs between the two samples defined by different maximal velocities by quite a large amount. This reflects the differences mentioned in Sect. 3.2 in the $N(\mathrm{Ia}) / N(\mathrm{CC})$ ratio as a function of the magnitude. We note that if we restrict ourselves to recent SN (right part of Fig. 8), the data from the 2 samples $\left(V_{\mathrm{HEL}}<2000\right.$, and $\left.5000 \mathrm{~km} \mathrm{~s}^{-1}\right)$ agree to within their respective error bars with each other (the error bars are however large). This suggests that misclassifications do play a role in our $N(\mathrm{Ia}) / N(\mathrm{CC})$ ratio and the slope of the relation is quite uncertain. However, in any cases, we do find a correlation.

ii) The same tendency is obtained for local metallicities. We note that, although the number of SNIa in each bin is rather small (see inserts for the case of $V_{\mathrm{HEL}}<2000 \mathrm{~km} \mathrm{~s}^{-1}$ ) the lifetimes of the progenitors of SNIa are quite long, in general, and thus we have not the problem described in the previous paragraphs for the SNIc/SNIb ratio (the more so, since the numbers of CCSN in each bin are quite substantial). Here again, very similar trends are obtained when we restrict ourselves to recent SN (even if large statistical error bars make the slope less significant, especially for the $V_{\mathrm{HEL}}<2000 \mathrm{~km} \mathrm{~s}^{-1}$ sample). The variation in the ratio across the entire range of metallicity is larger than the uncertainties (mentioned in Sect. 2) due to the Shaw (1979) effect, or the presence of sub-luminous SN. We conclude then that the trend of increasing $\mathrm{SNIa} / \mathrm{CCSN}$ ratio with metallicity is likely to be real, although the relation between the SNIa/CCSN ratio and metallicity still carries large uncertainties. 


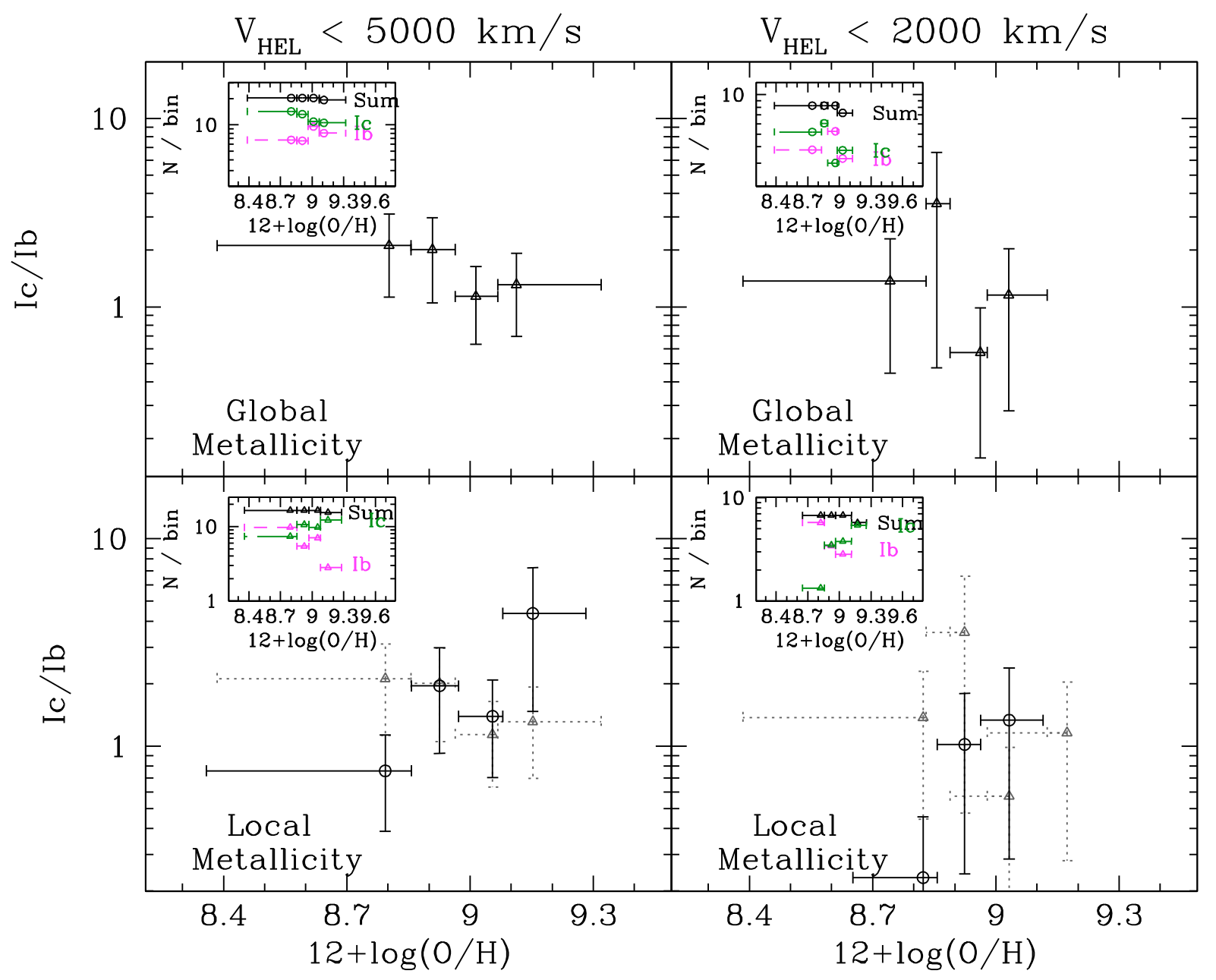

Fig. 7. Symbols with solid errror bars: number ratios of SNIc/SNIb as a function of global galaxian metallicity (top, obtained from the magnitudemetallicity relation of Fig. 4) and of local metallicity (bottom, obtained from Eq. (6)), for the conservative $V_{\mathrm{HEL}}<2000 \mathrm{~km} \mathrm{~s}^{-1}$ sample (right) and the $V_{\mathrm{HEL}}<5000 \mathrm{~km} \mathrm{~s}^{-1}$ sample (left, better statistics). Inserts in all panels display corresponding numbers of SN in each bin (chosen such as the total numbers are approximately equal). Triangles with dotted error bars in lower panels are the same as those with solid error bars in the upper panels and are displayed for comparison with local metallicity results.

\section{Discussion}

\subsection{The $N(I b c) / N(I I)$ ratio}

The variation in the $N(\mathrm{Ibc}) / N(\mathrm{II})$ ratio with metallicity has been quite well established (PB03; Prieto et al. 2008, and this work). It has been equally well established that non-rotating single star models can reproduce neither the observed trend nor the average value of that ratio. This is clearly seen in Fig. 9, where we plot the results of Eldridge et al. (2008) (long dashed curve).

As suggested in PB03, single star models with rotation are in this respect promissing. In Fig. 9, we present the results of these models from the Geneva group (Maeder \& Meynet 2004, dot-dashed curve; Meynet et al. 2008, dotted curve). They are both obtained for a power-law IMF of slope $x=-1.35$. Their behaviour is compatible with observational data, especially if observational error bars are taken into account.

An even more robust fit to the data is obtained by the binary star models of Eldridge et al. (2008). This result is surprising, since it is not a priori obvious how metallicity can affect to such extent the evolution of stars in binaries. Eldridge et al. (2008) argue that most of the hydrogen envelope is removed by binary interaction in the case of close binary systems, and that metallicity-dependent stellar winds remove the remainder.
In view of those results, it appears difficult to decide whether stellar rotation or binary evolution produces the observed trend. Theoretical uncertainties are quite important in both cases (but certainly more so in the case of binary evolution), making it premature to draw firm conclusions. It may well be that both factors contribute to the observed trend.

Stellar models predict the metallicity dependence of $M_{\mathrm{Ibc}}$, the minimum mass for a single star to lose its hydrogen envelope. Using a stellar MF allows one to calculate the resulting $N(\mathrm{Ibc}) / N(\mathrm{II})$ ratio versus metallicity, as e.g., in Fig. 9. Inversely, observed $N(\mathrm{Ibc}) / N(\mathrm{II})$ ratio versus metallicity can be used to infer $M_{\mathrm{Ibc}}$ versus metallicity. This was done for the first time in PB03, who predicted the metallicity dependence of $M_{\mathrm{Ibc}}$ on the basis of then available data for $N(\mathrm{Ibc}) / N(\mathrm{II})$ versus metallicity. In Fig. 10, we present the result of both PB03 (thin solid curve) and our new evaluation (thick solid curve) for a slope $x=-1.35$ of the IMF. The two curves are close to each other and not very different from the theoretical predictions of Meynet et al. (2008, dotted curve) at low metallicities. We note that to compare the results of Meynet et al. (2008), expressed as a function of $Z / Z_{\odot}$, to our own, expressed as a function of $\mathrm{O} / \mathrm{H}$, we assume that the solar oxygen abundance is $\log (\mathrm{O} / \mathrm{H})_{\odot}+12=8.8$; this value is close to that determined for 


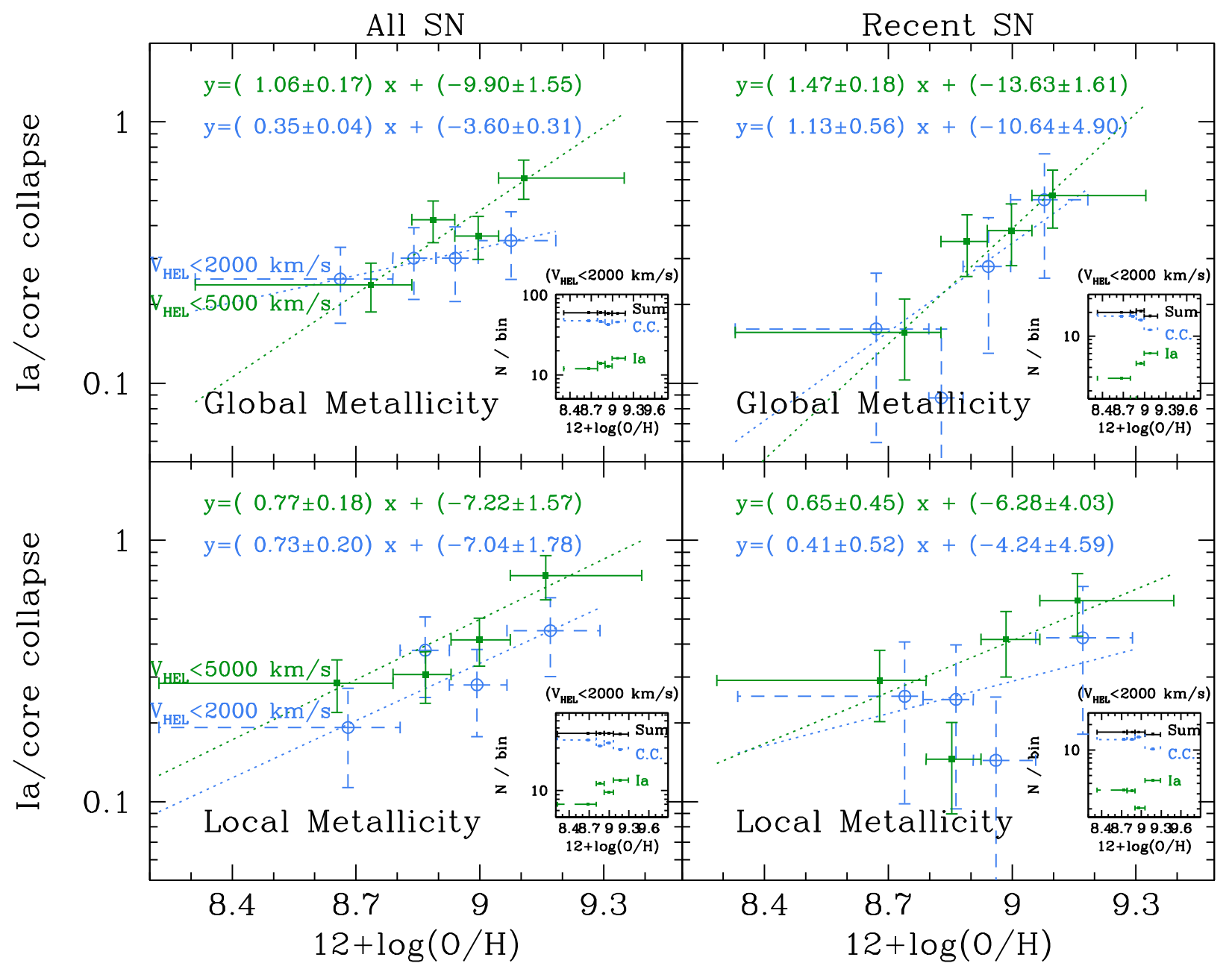

Fig. 8. Number ratio of SNIa/CCSN as a function of global (top) and local (bottom) galaxian metallicity. Data are displayed (left panels) for our two samples adopting various maximal heliocentric velocities $V_{\mathrm{HEL}}$ of the host galaxies: $2000 \mathrm{~km} \mathrm{~s}^{-1}$ (dashed), and $5000 \mathrm{~km} \mathrm{~s}^{-1}$ (solid). The same is shown in the right panels but only for recent SN (events that occured during and after 2000).

the Sun $\log (\mathrm{O} / \mathrm{H})_{\odot}+12=8.76 \pm 0.007$ by Caffau et al. (2008). At high metallicities observationally determined $M_{\mathrm{Ibc}}$ is systematically lower than the predictions of Meynet et al. (2008) but by only a couple of solar masses. We Note that the theoretical predictions of $M_{\mathrm{Ibc}}$ are independent of the IMF, while the empirical determinations do depend on it: a steeper IMF would produce a $M_{\text {Ibc }}$ versus metallicity curve lower by a few solar masses than the one shown in Fig. 10. In contrast, the theoretical $N(\mathrm{Ibc}) / N(\mathrm{II})$ ratio versus metallicity does depend on the IMF: a steeper IMF would produce $N(\mathrm{Ibc}) / N(\mathrm{II})$ ratios lower than depicted in Fig. 9.

Given the various uncertainties, our estimates of the minimum mass of SNIbc at solar metallicity are in fairly good agreement with empirical estimates for the minimum mass of WN stars in the Milky Way, which lies in the 20-25 $M_{\odot}$ range (Massey et al. 2001; Massey 2003; Crowther 2007).

\subsection{The $N(I c) / N(I b)$ ratio}

In the case of the $N(\mathrm{Ic}) / N(\mathrm{Ib})$ ratio, the observational situation is not at present clear, since different trends are obtained as a function of global and local metallicity; we argued in the previous section that small number statistics are at the origin of this dichotomy. One may only determine a global value of $N(\mathrm{Ic}) / N(\mathrm{Ib}) \sim 1.6$, i.e., there are about $50 \%$ more SNIc than SNIb. Fryer et al. (2007) find that this high ratio is “ ... against intuition in the single star case and it may be a further argument in favor of binary origin for SNIc". However, a simple evaluation of the limiting masses on the basis of global observations and for a Salpeter IMF with slope $x=-1.35$ gives $M_{\mathrm{Ib}}=24 M_{\odot}$ and $M_{\mathrm{Ic}}=31 M_{\odot}$ (Arbutina 2007), i.e. it is easy to obtain the observed ratio if it is assumed that SNIb originate in stars of a limited range of masses (between 24 and $31 M_{\odot}$, with more massive stars exploding as SNIc).

From the theoretical point of view, there is a dearth of predictions for $N(\mathrm{Ic}) / N(\mathrm{Ib})$ even for the single star case. It is generally assumed that SNIc originate from more massive stars than SNIb. However, there is no agreement on the amount of He left in the envelope for a star to explode as SNIc. Moreover, although it is generally agreed that the most massive stars end up in black holes, it is not clear what happens when a black hole is formed: is a bright emission of radiation still obtained, or is the explosion underluminous or does it even fail (see e.g. Fryer et al. 2007, and references therein)? In the last case, the upper part of the IMF would not contribute to SNIc and the $N(\mathrm{Ic}) / N(\mathrm{Ib})$ ratio could be small.

We present in Fig. 10 for illustration purposes three curves for the mass limit $M_{\text {Ic }}$ as a function of metallicity, on the basis of various assumptions about the true observational trend. We assume that the stellar IMF extends up to $100 M_{\odot}$ and that the results of this work for $M_{\mathrm{Ib}}$ (thick solid curve in Fig. 10) are 


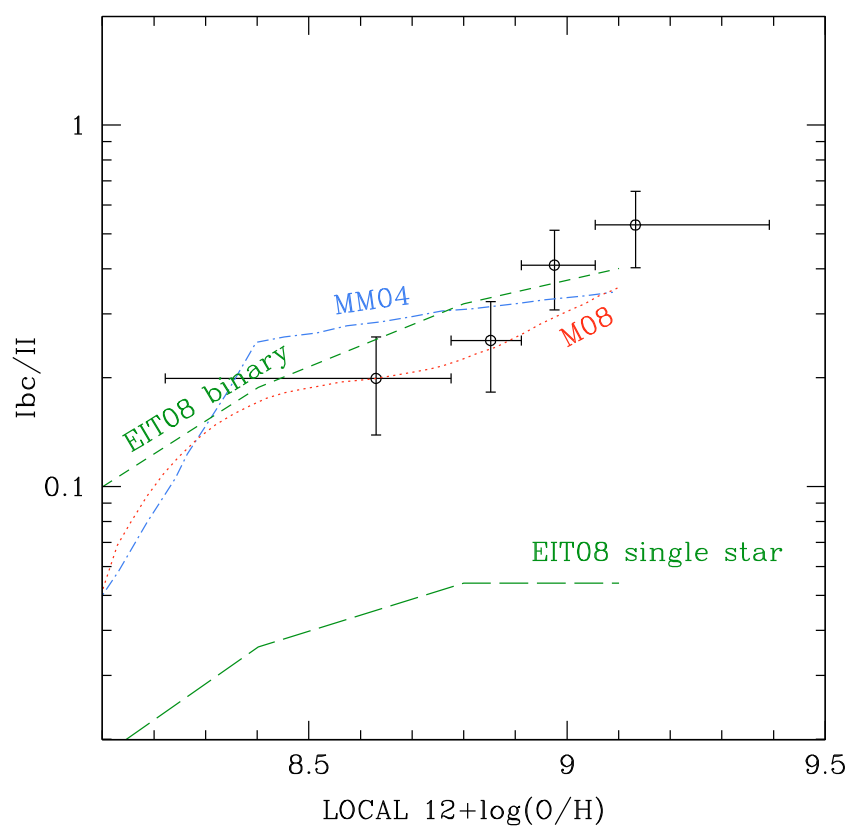

Fig. 9. Number ratio of SNIbc/SNII as a function of local galaxian metallicity and comparison to single star rotating models of Maeder \& Meynet (2004, MM04, dot-dashed) and of Meynet et al. (2008, M08, dotted), single non-rotating stellar models of Eldridge et al. (2008, EIT08, long dashed) and binary non-rotating stellar models of Eldridge et al. (2008, EIT08, short dashed).

correct. The slope of the IMF plays little role in the resulting $M_{\text {Ic }}$ and we consider here only the case of $x=-1.35$ (Salpeter slope). We proceed by considering three possible cases for the variation in $N(\mathrm{Ic}) / N(\mathrm{Ib})$ ratio with metallicity, on the basis of the results presented in Fig. 7:

i) $\quad N(\mathrm{Ic}) / N(\mathrm{Ib})=$ const. $=1.6$ (upper left panel in Fig. 7). This leads to the lowest of the three curves for $M_{\text {Ic }}$ in Fig. 10 (short-dashed curve): the curve runs almost parallel to the one for $M_{\mathrm{Ib}}$, at a "distance" of a 6-10 $M_{\odot}$, i.e., SNIb are produced only for a limited range of stellar masses from 45 to $55 M_{\odot}$ at the lowest metallicities and from 20 to $26 M_{\odot}$ at the highest metallicities.

ii) $N(\mathrm{Ic}) / N(\mathrm{Ib})=Z / Z_{\odot}$ at all metallicities (lower left panel of Fig. 7, trend extrapolated to lower than solar metallicities). In that case, we obtain the highest lying curve in Fig. 10 (long-dashed). At low metallicities, the ratio is low and the limiting mass $M_{\mathrm{Ic}}$ is as high as $75 M_{\odot}$, while at high metallicities we recover the results of the previous case.

iii) $N(\mathrm{Ic}) / N(\mathrm{Ib})=Z / Z_{\odot}$ at $Z>Z_{\odot}$ and $N(\mathrm{Ic}) / N(\mathrm{Ib})=1$ for $Z<Z_{\odot}$. This case leads to a curve intermediate between the two previous ones (dot-dashed in Fig. 10).

The derived curves confirm the findings of Arbutina (2007) on the basis of global SN ratios, namely that it is possible to have both SNIb and SNIc solely from single star evolution; this suggests that the concern expressed in Fryer et al. (2007) was unfounded. If the $N(\mathrm{Ic}) / N(\mathrm{Ib})$ ratio is $\sim 2$ at high metallicities, then the range of masses producing SNIb is rather limited. This is also found in the single star models with rotation of Meynet et al. (2008), although no quantitative predictions are given by these authors. The wide spacing between the curves of $M_{\text {Ic }}$ corersponding to cases (i) and (iii) in Fig. 10 suggests that substantial effort is required to ascertain the true metallicity dependence of $N(\mathrm{Ic}) / N(\mathrm{Ib})$ ratio with larger data sets.

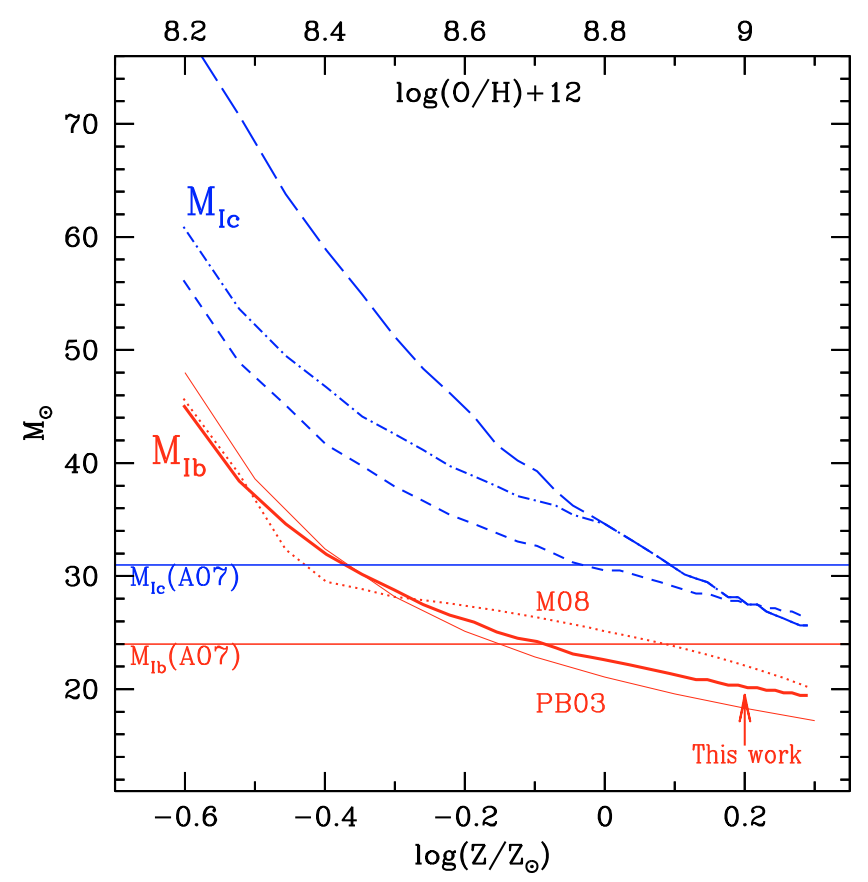

Fig. 10. Minimum mass for single stars becoming SNIb or SNIc as a function of metallicity, according to theoretical and empirical evaluations. The two horizontal lines are from Arbutina (2007), based on measured global SNIbc/CCSN and SNIc/SNIb ratios. The three lower curves are for $M_{\mathrm{Ib}}$, from theoretical (Meynet et al. 2008, dotted) and empirical evaluations (PB03, thin solid and this work thick solid). Finally, the three upper curves are for $M_{\mathrm{Ic}}$ from this work, assuming that the $M_{\mathrm{Ib}}$ versus metallicity curve of this work is correct and that the IMF extends up to $100 M_{\odot}$. The three curves are obtained then assuming that: i) the SNIc/SNIb ratio is 1.6, as in upper left panel in Fig. 7 (short dashed), ii) the SNIc/SNIb ratio depends on metallicity as in lower left panel in Fig. 7 extrapolated below the lowest observed metallicity (long dashed), and iii) the SNIc/SNIb ratio depends on metallicity only for $Z>Z_{\odot}$ and remains equal to unity below solar (dot-dashed). The two metallicity scales match each other for $\log (\mathrm{O} / \mathrm{H})_{\odot}+12=8.8$.

\subsection{The $N(l a) / N(C C)$ ratio}

In Sect. 3.2 we provide an explanation of the observed variation in $N(\mathrm{Ia}) / N(\mathrm{CC})$ ratio with global metallicity. Here we provide a similar argument for the observed variation in the $N(\mathrm{Ia}) / N(\mathrm{CC})$ ratio with local metallicity. For a star-forming galactic disk, Eq. (5) can be rewritten in terms of the local surface densities of stars $\Sigma_{*}(R)$ and star formation rate $\Psi(R) \propto \Sigma_{\mathrm{g}}^{k}(R)$, where $\Sigma_{\mathrm{g}}(R)$ is the gas surface density at galactocentric distance $R$ and $k=1.4$ is the coefficient in the Kennicutt (1998) empirical "star formation law", as

$\frac{N(\mathrm{Ia})}{N(\mathrm{CC})}=A+B \frac{\Sigma_{*}}{\Sigma_{\mathrm{g}}^{k}}$

where all variables depend on radial distance $R$. Stellar profiles in galactic disks are usually fitted with exponentials of scalelength $R_{*}$, i.e., $\Sigma_{*}(R) \propto \exp \left(-R / R_{*}\right)$. Corresponding gaseous profiles are always much flatter than stellar ones and if fitted by exponentials they would be $\Sigma_{\mathrm{g}}(R) \propto \exp \left(-R / R_{\mathrm{g}}\right)$ with $R_{\mathrm{g}}>2 R_{*}$. In the case of the Milky Way, for instance, one has $R_{*} \sim 2.5 \mathrm{kpc}$ and $R_{\mathrm{g}} \sim 8 \mathrm{kpc}$; further examples for external disk galaxies can be found in e.g., Boissier et al. (2003). Equation (7) is then rewritten as

$\frac{N(\mathrm{Ia})}{N(\mathrm{CC})}=A+C \exp \left(-\frac{R_{\mathrm{g}}-k R_{*}}{R_{\mathrm{g}} R_{*}} R\right)$, 

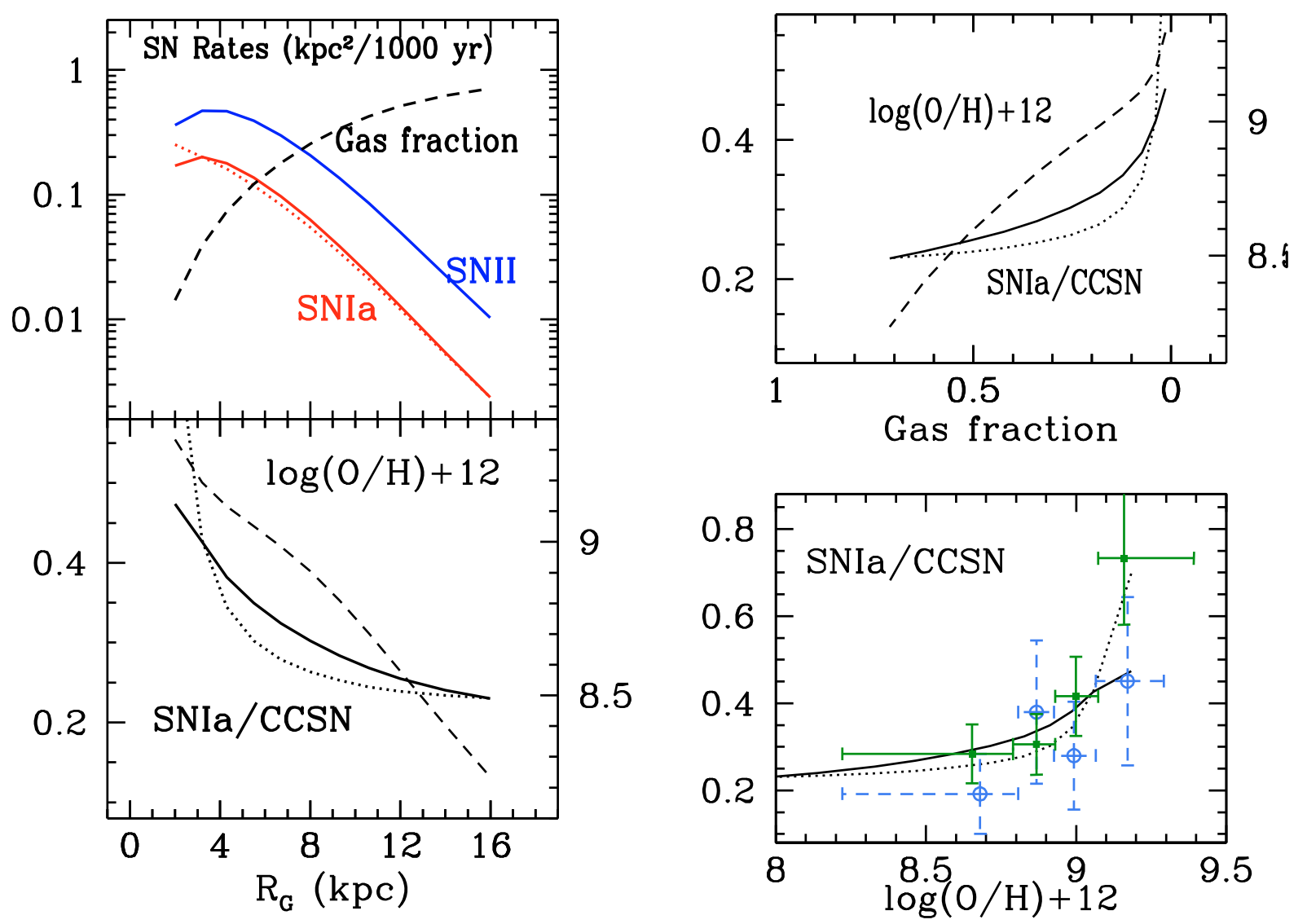

Fig. 11. Illustration of the variation in SNIa/CCSN ratio as a function of metallicity, with a realistic model of galactic evolution (from Boissier \& Prantzos 1999). Top left: rates of CCSN (solid) and SNIa (solid: model, dotted: analytical) and gas fraction (dashed) as a function of galactocentric radius. Bottom left: SNIa/CCSN ratio (solid: model, dotted: analytical) and metallicity profile (dashed, right vertical axis). Top right: SNIa/CCSN ratio (solid: model, dotted: analytical) and metallicity (dashed, right vertical axis). Bottom right: SNIa/CCSN ratio (solid: model, dotted: analytical) as a function of metallicity; comparison is made to the data for local metallicity of Fig. 8 (bottom-left).

where $C$ is a new constant. For reasonable values of $k(<2)$, it can clearly be seen that, Eq. (8) is a decreasing function of radius $R$. Thus, in galactic disks the $N(\mathrm{Ia}) / N(\mathrm{CC})$ ratio is expected to increase towards the inner galaxy, i.e., to be correlated with metallicity, as observed.

We illustrate this behaviour in Fig. 11, where we plot the relevant quantities for the case of the Milky Way disk. All curves are obtained from an updated successful model of the Galactic disk (from Boissier \& Prantzos 1999). For the SNIa rate, the formalism by Greggio \& Renzini (1983) is adopted in the model and the corresponding results are displayed with solid curves in all panels. We also apply the simple analytic expression of Eq. (8) and the corresponding results are plotted with dotted curves. Figure 11 displays the present-day $(T=12.5 \mathrm{Gyr})$ radial profiles of CCSN, SNIa, and gas fraction (top left panels), the profiles of $N(\mathrm{Ia}) / N(\mathrm{CC})$ ratio and oxygen (bottom left panels), and shows clearly that both $N(\mathrm{Ia}) / N(\mathrm{CC})$ and metallicity increase at lower gas fractions, i.e., in the inner disk (top right panel). Finally, in the bottom right panel we show that the resulting $N(\mathrm{Ia}) / N(\mathrm{CC})$ versus metallicity relation compares favorably with the data of Fig. 8; the analytical prescription for the SNIa rate leads to a more steeply rising $N(\mathrm{Ia}) / N(\mathrm{CC})$ ratio with metallicity than the prescription of Greggio \& Renzini (1983), but in both cases the agreement with observations is satisfactory. Obviously, an increase in the observational sample of SN will allow us in the future to reduce the size of the error bars and to constrain prescriptions for the SNIa rate.

\section{Summary}

We have derived relationships between the ratios of various SN types and metallicity of host galaxies. For that purpose, we have determined either global metallicities (reflecting the composition at radius $R=0.4 R_{25}$ ) or local ones, i.e., at the position of the $\mathrm{SN}$ inside the host galaxy. In the former case, we used the well known metallicity-magnitude relationship, a technique applied already in PB03 (albeit with a smaller SN sample). In the latter case, we assumed that galaxian metallicity gradients are approximately constant when expressed in $\operatorname{dex} / R_{25}$; this method is applied for the first time, to our knowledge, to the determination of local metallicities in disks and appears to be quite promising. We performed a number of tests, defining several samples and using different values for the abundance gradients, to ensure that the observed trends were not biased.

We find that $N(\mathrm{Ibc}) / N(\mathrm{II})$ ratio increases with both global galaxy metallicity (as already found in PB03 and in Prieto et al. 2008) and local one. Our study reduces the size of the error bars of previous works considerably. We now consider this result to be established. The variation in $N(\mathrm{Ibc}) / N(\mathrm{II})$ with metallicity is larger than the changes that could cause any biases we can think of, and the trend is consistently obtained for almost all our samples, e.g., with various assumptions or limits on $V_{\mathrm{HEL}}$, with the exception of the $V_{\mathrm{HEL}}<2000 \mathrm{~km} \mathrm{~s}^{-1}$ sample in which the ratio for the higher global metallicity bin is lower than expected for such a trend. We have discussed the results in terms of either 
single star models with rotation or binary evolution models. In view of observational and theoretical uncertainties, which are certainly larger for binary evolution than for single stars, we find it difficult to chose between the two possibilities. Assuming that only single stars produce SNIbc, we derive the empirical $M_{\mathrm{Ibc}}$ versus metallicity relation and find it to be compatible with the one obtained with the latest models of the Geneva group (Meynet et al. 2008).

We study the $N(\mathrm{Ic}) / N(\mathrm{Ib})$ ratio and we find it consistent with being constant with global metallicity but increasing with local metallicity. We attribute this difference to small number statistics and we believe that larger SN samples in the future will allow us to distinguish between the two possibilities. Nevertheless, we derive the empirical $M_{\text {Ic }}=f(Z)$ relation assuming again that only single stars produce SNIb and SNIc. We find that this assumption leads to SNIb being produced within a relatively limited range of stellar masses, as found in rotating star models of Meynet et al. (2008). We show that the single star channel can justify $N(\mathrm{Ic}) / N(\mathrm{Ib})$ ratios as high as 2 and we disagree in this respect with Fryer et al. (2007) in their claim that such a high ratio favours a binary formation channel for SNIc.

Finally, for the first time, we find an unexpected correlation between the ratio $N(\mathrm{Ia}) / N(\mathrm{CC})$ and metallicity, both globally and locally. Although the precise values of the slope and intersect may be affected by the existing large uncertainties (various samples provide different results), the trend is statistically secure (the null hypothesis has a probability of only $7 \%$ using local metallicity and the conservative $V_{\mathrm{HEL}}<2000 \mathrm{~km} \mathrm{~s}^{-1}$ sample). We argue that this is not a causal relationship, in contrast to the previous cases; instead, both $N(\mathrm{Ia}) / N(\mathrm{CC})$ and metallicity are higher in regions of lower gas fractions (or lower specific star formation rates). We develop this argument analytically and we illustrate it with a quantitative application to the case of the Milky Way disk.

Comparing this situation to that only $\sim 6$ years ago, we find that the increase in the SN sample size since PB03, and the use of local metallicities rather than global ones has allowed us to establish the strong likelihood of the $N(\mathrm{Ibc}) / N(\mathrm{II})$ versus metallicity trend. We expect then that a similar increase in the future will allow one to establish reliably the $N(\mathrm{Ic}) / N(\mathrm{Ib})$ versus metallicity trend and to probe with greater accuracy the details of the SNIa rate.

Acknowledgements. We thank S. Basa and T. Zhang for useful discussions. We thank the referee, S. Smartt for his detailed review of the paper and his suggestions. We acknowledge the usage of the HyperLeda database (http://leda.univ-lyon1.fr), as well of the PadovaAsiago supernova catalogue, from the Padova-Asiago Supernova Group (http://web.pd.astro.it/supern/).

\section{References}

Anderson, J. P., \& James, P. A. 2008, MNRAS, 390, 1527

Arbutina, B. 2007, Int. J. Mod. Phys. D, 16, 1219

Aubourg, E., Tojeiro, R., Jimenez, R., et al. 2008, A\&A, 492, 631

Barbon, R., Buondí, V., Cappellaro, E., \& Turatto, M. 1999, A\&AS, 139, 531

Boissier, S., \& Prantzos, N. 1999, MNRAS, 307, 857

Boissier, S., \& Prantzos, N. 2000, MNRAS, 312, 398

Boissier, S., Boselli, A., Prantzos, N., \& Gavazzi, G. 2001, MNRAS, 321, 733

Boissier, S., Prantzos, N., Boselli, A., \& Gavazzi, G. 2003, MNRAS, 346, 1215

Bresolin, F. 2007, ApJ, 656, 186

Bresolin, F., Garnett, D. R., \& Kennicutt, R. C., Jr. 2004, ApJ, 615, 228

Bressan, A., Della Valle, M., \& Marziani, P. 2002, MNRAS, 331, L25

Caffau, E., Ludwig, H.-G., Steffen, M., et al. 2008, A\&A, 488, 1031

Cappellaro, E., Turatto, M., Benetti, S., et al. 1993, A\&A, 273, 383

Crowther, P. A. 2007, ARA\&A, 45, 177

Eldridge, J. J., Izzard, R. G., \& Tout, C. A. 2008, MNRAS, 384, 1109

Fryer, C. L., Mazzali, P. A., Prochaska, J., et al. 2007, PASP, 119, 1211

Garnett, D. R. 2002, ApJ, 581, 1019

Garnett, D. R., Shields, G. A., Skillman, E. D., Sagan, S. P., \& Dufour, R. J. 1997, ApJ, 489, 63

Greggio, L., \& Renzini, A. 1983, A\&A, 118, 217

Hakobyan, A. A. 2008, Astrophys., 51, 69

Hamuy, M. 2002, in Core Collapse of Massive Stars, ed. C. L. Fryer (Dordrecht: Kluwer)

Heger, A., Fryer, C. L., Woosley, S. E., Langer, N., \& Hartmann, D. H. 2003, ApJ, 591, 288

Henry, R. B. C., \& Worthey, G. 1999, PASP, 111, 919

Kelly, P. L., Kirshner, R. P., \& Pahre, M. 2008, ApJ, 687, 1201

Kennicutt, R. C., Jr. 1998, ApJ, 498, 541

Maeder, A., \& Meynet, G. 2004, A\&A, 422, 225

Mannucci, F., Della Valle, M., Panagia, N., et al. 2005, A\&A, 433, 807

Massey, P. 2003, ARA\&A, 41, 15

Massey, P., DeGioia-Eastwood, K., \& Waterhouse, E. 2001, AJ, 121, 1050

Meynet, G., \& Maeder, A. 2003, A\&A, 404, 975

Meynet, G., Chiappini, C., Georgy, C., et al. 2008 [arXiv:0810.0652]

Modjaz, M., Kawley, L., Kirshner, R. P., et al. 2008, AJ, 135, 1136

Paturel, G., Petit, C., Prugniel, P., et al. 2003, A\&A, 412, 45

Prantzos, N., \& Boissier, S. 2000, MNRAS, 313, 338

Prantzos, N., \& Boissier, S. 2003, A\&A, 406, 259

Prieto, J. L., Stanek, K. Z., \& Beacom, J. F. 2008, ApJ, 673, 999

Richardson, D., Branch, D., Casebeer, D., et al. 2002, AJ, 123, 745

Richardson, D., Branch, D., \& Baron, E. 2006, AJ, 131, 2233

Scannapieco, E., \& Bildsten, L. 2005, ApJ, 629, L85

Shaw, R. L. 1979, A\&A, 76, 188

Smartt, S. J., Eldridge, J. J., Crockett, R. M., \& Maund, J. R. 2009, MNRAS, 395,1409

Sullivan, M., Le Borgne, D., Pritchet, C. J., et al. 2006, ApJ, 648, 868

Tremonti, C. A., Heckman, T. M., Kauffmann, G., et al. 2004, ApJ, 613, 898

Turatto, M. 2003, in Supernovae and GRBs, v. 598 of Lecture Notes in Physics,

ed. K. Weiler (Springer-Verlag), 21

van den Bergh, S. 1997, AJ, 113, 197

van den Bergh, S., Li, W., \& Filippenko, A. V. 2005, PASP, 117, 773

van Zee, L., Salzer, J. J., Haynes, M. P., O’Donoghue, A. A., \& Balonek, T. J. 1998, AJ, 116, 2805

Zaritsky, D., Kennicutt, R. C., Jr., \& Huchra, J. P. 1994, ApJ, 420, 87 